Liu Di (Orcid ID: 0000-0003-3241-1421)

Howell Kate (Orcid ID: 0000-0001-6498-0472)

\title{
Community succession of the grapevine fungal microbiome in the annual growth cycle
}

Running title

The core fungal microbiome of grapevines

Di Liu and Kate Howell*

School of Agriculture and Food, Faculty of Veterinary and Agricultural Sciences, University of Melbourne Parkville 3010 Australia 7

*Corresponding author. khowell@unimelb.edu.au

This is the author manuscript accepted for publication and has undergone full peer review but has not been through the copyediting, typesetting, pagination and proofreading process, which may lead to differences between this version and the Version of Record. Please cite this article as doi: 10.1111/1462-2920.15172

This article is protected by copyright. All rights reserved. 


\section{Summary}

Microbial ecology and activity in wine production influences grapevine health and productivity, conversion of sugar to ethanol during fermentation, wine aroma, wine quality and distinctiveness. Fungi in the vineyard ecosystem are not well described. Here, we characterised the spatial and temporal dynamics of fungal communities associated with the grapevine (grapes, flowers, leaves, and roots) and soils over an annual growth cycle in two vineyards to investigate the influences of grape habitat, plant developmental stage (flowering, fruit set, veraison, and harvest), vineyards, and climatic conditions. Fungi were influenced by both the grapevine habitat and plant development stage. The core microbiome was prioritised over space and time, and the identified core members drove seasonal community succession. The developmental stage of veraison, where the grapes undergo a dramatic change in metabolism and start accumulating sugar, coincided with a distinct shift in fungal communities. Co-occurrence networks showed strong correlations between the plant microbiome, the soil microbiome, and weather indices. Our study describes the complex ecological dynamics that occur in microbial assemblages over a growing season and highlight succession of the core community in vineyards.

\section{Keywords: grapevine, fungal ecology, plant development, core microbiome, climate}

\section{Introduction}

The grapevine (Vitis vinifera) is naturally colonised by complex and diverse microorganisms, such as bacteria, filamentous fungi, and yeasts (Barata et al., 2012; Stefanini and Cavalieri, 2018), which substantially modulate vine health, growth, and productivity (Gilbert et al., 2014; Müller et al., 2016). Microbes originate from the surrounding environment (including soil, air, precipitation, animal vectors, native forests) and colonise both exterior (epiphytes) and interior (endophytes) (Goddard et al., 2010; Stefanini et al., 2012; Lam and Howell, 2015; Zarraonaindia et al., 2015; Morrison-Whittle and Goddard, 2018). This microbiota can be transferred to the grape must (or juice) and thus have a profound influence on wine composition, flavour, 
aroma, and quality (Ciani et al., 2010; Barata et al., 2012; Morrison-Whittle and Goddard, 2018).

Saccharomyces yeasts (primarily Saccharomyces cerevisiae) and lactic acid bacteria (predominantly

Oenococcus oeni) are primary drivers of wine fermentation and to form wine flavour and aroma (Swiegers et al., 2005). Beyond these species, our knowledge about the microbial ecology associated with grapevines and its role in the vineyard ecosystem is limited.

Recent studies posit the existence of geographically structured microbiota that contribute to the expression of regional distinctiveness of agricultural products, specifically, known as "terroir" in viticulture [reviewed by Liu et al. (2019)]. Significant biogeographic patterns are generally observed at scales of hundreds to thousands of kilometres (Gayevskiy and Goddard, 2012; Bokulich et al., 2014; Taylor et al., 2014; Knight and Goddard, 2015), and influenced by multiple factors, such as climate and topography, soil properties, and even local anthropogenic practices (Bokulich et al., 2014; Pinto et al., 2014; Burns et al., 2015; Zarraonaindia et al., 2015; Jara et al., 2016; Portillo et al., 2016; Morrison-Whittle and Goddard, 2017). Microbial spatial pattern analyses have mainly been conducted at large scales and focused on studying grapes and must, the communities present at the start of fermentation, and the soil, but the nature and the importance of microbial ecology within vineyards towards defining terroir, is largely unknown.

Metagenomics tools have been actively used to map the plant microbiome, in particular the phyllosphere and the rhizosphere (Turner et al., 2013; Müller et al., 2016). In viticulture, many studies have been dedicated to characterising grapevine-associated bacteria and their relevant importance on plant growth and winemaking processes (Zarraonaindia et al., 2015; Marasco et al., 2018). Zarraonaindia et al. (2015) investigated the spatial and temporal dynamics of the bacterial microbiota associated with grapevine organs of Merlot cultivars in New York state, and revealed significant differences among grapevine habitats and the soil, although the latter serving as a source reservoir for the former. The endophytic fungi are diverse and structured in 
rhizocompartments and the vascular system (Deyett and Rolshausen, 2020). Fungal communities across multiple compartments have not been systematically investigated. As described in other plant systems, microbiome composition is not temporally stable but change in response to plant development (Mougel et al., 2006; Jumpponen and Jones, 2010; Chaparro et al., 2014; Copeland et al., 2015; Grady et al., 2019) and can fundamentally affect the health and productivity of plants. Core microbiomes are commonly defined based on abundance-occupancy distributions describing microbes which are highly abundant and ubiquitous occurring the habitat/plant species over time and are hypothesised to reflect underlying functional relationships with the host (Lundberg et al., 2012; Shade and Stopnisek, 2019). Identifying members and characteristics of core microbiomes, which are expected to play pivotal roles in organising the dynamics of resident microbiomes, will be the key to optimising interactions between plants and indigenous microorganisms and increasing resource-efficiency and stress-resistance of agroecosystems (Toju et al., 2018). In grapevines, however, this remains to be shown.

In the present study, we sampled microbial communities associated with Pinot Noir grapevines from two vineyards within the same grape growing region to provide insights into fungal ecology in the vineyard. Using internal transcribed spacer (ITS) amplicon sequencing to characterise fungal communities, we disentangled the influences of geographic location (between two vineyards), grapevine habitat (soil, roots, leaves, flowers and grapes), grapevine developmental stage (flowering, fruit set, veraison, harvest), and climatic conditions on the fungal diversity, composition, and structure from root zone soil, roots, leaves, and grapes or flowers. Specifically, we aimed to prioritise the core microbiome over space and time, and further confirm the role of this core community to structure seasonal community succession based on Random Forest supervised learning models. The influence of the changing environment was elucidated with network analysis. We show that fungal communities differ according to grapevine habitats and developmental stages, with little geographic influences at this scale $(5 \mathrm{~km})$. The core microbiome correlates closely with solar radiation and water status 
during the annual growth cycle and drives seasonal community succession. Combining our study with studies in other grape growing areas will expand our knowledge of the core microbiome associated with grapevine across different soil types and environmental conditions to allow optimisation of the growth of this important economic crop.

\section{Results}

\section{Fungal communities vary by grapevine habitat within vineyards}

A total of 4,786,543 ITS high-quality sequences were generated from all samples ( $\mathrm{n}=160)$, which were clustered into 2,286 fungal OTUs with a threshold of 97\% pairwise identity. No OTUs were found to be common across all the samples. Among all the OTUs identified in the present study, $62.90 \%$ were detected in more than one grapevine habitat (root zone soil, root, leaf, flower and grape), of which 54 OTUs were shared across sample types (Fig. 1A). The soil contained the most OTUs and of these, 37.67\% of OTUs found in soils were also associated with the plant, with $32.75 \%$ of OTUs shared between soils and roots which was the highest portion of OTUs shared between soil and plant organs (5.86\% between soils and leaves, $11.58 \%$ between soils and flowers, 7.95\% between soils and grapes). Each plant organ shared the majority of fungal taxa with other organs and the soil. Some OTUs were unique for specific organs, with low occurrence frequencies. 79 OTUs found in grapes were observed specific for this organ (Fig. 1A) with a low occurrence frequency $\leq 10 \%$, accounting for $28.11 \%$ of total grape-associated OTUs; likewise, the organ-specific OTU portion was $9.11 \%$ for flowers (frequency $\leq 30 \%$ ), $8.24 \%$ for leaves (frequency $\leq 2.5 \%$ ), and $8.53 \%$ for roots (frequency $\leq 7.5 \%$ ). Ascomycota was the most abundant phylum in the vineyard habitats comprising $77.69 \%$

of all sequences, followed by Basidiomycota and Mortierellomycota in the plant, and Mortierellomycota and Basidiomycota in the soil, respectively (data not shown). The major fungal genera were Aureobasidium, Cladosporium, Epicoccum, Mortierella, Cryptococcus, Debaryomyces, Saccharomyces, Mycosphaerella, Lophiostoma, Alternaria, and Penicillium, with relative abundance of more than 1.0\% across all samples (Fig. 
1B). Some major taxa associated with plants, such as Aureobasidium, Debaryomyces, Saccharomyces and Mycosphaerella, were present in soil samples at very low abundances (<0.05\%). The genus Mortierella had the highest relative abundance (26.00\%) in the soil, and showed lower abundances in the plant (Fig. 1B).

Grapevine-associated fungi varied with vine habitat (root zone soil, root, leaf, flower and grape) and exerted significant differences in both community diversity ( $\alpha$-diversity, Shannon index; ANOVA, F $(4,155)=5.206, p$ $<0.001$ ) and dissimilarity ( $\beta$-diversity, Bray-Curtis distance; PERMANOVA, $\mathrm{R}^{2}=0.271, p<0.001$ ) independent of phenological development stage (Table 1). This trend was more distinct with an improved coefficient of determination $\left(\mathrm{R}^{2}\right)$ within a certain stage (Table 1). Geographic location only weakly impacted microbial diversity and composition, except for fungal diversity associated with roots, which was significantly impacted by vineyard (ANOVA, $\left.\mathrm{F}_{(1,38)}=5.878, p<0.01\right)$ (Table 1). Principal coordinate analysis $(\mathrm{PCoA})$ showed the habitat-specific patterns (95\% confidence interval) of fungal communities, with $47.4 \%$ of total variance explained by the first two principal coordinate (PC) axes using the harvest stage as an example (Fig. 1C). Linear discriminant analysis (LDA) effect size (LEfSe) analysis further confirmed that these patterns related to significant associations (Kruskal-Wallis sum-rank test, $\alpha<0.05$ ) between fungal taxa and sample types (Fig. 1D). At harvest, Mortierellomycota (notably Mortierella amoeboidea), Agaricomycetes, Xylariales (including Amphisphaeriaceae), Cucurbitariaceae (including Pyrenochaetopsis), Didymella pedeiae, Exophiala equina and Penicillium onobense were observed with higher abundances in the soil. For grapevine habitats, Saccharomycetaceae (notably fermentative yeast S. cerevisiae), Cryptococcus saitoi (Basidiomycota yeasts), Dothioraceae (including Aureobasidium) and Mycosphaerellaceae (including Mycosphaerella) were significantly more abundant in roots than in other habitats, whereas Saccharomycetes (including Debaryomyces) and Fusarium denticulatum were more abundant in leaves, and Ascomycota (notably Cladosporium) in grapes. 


\section{Temporal fungal community dynamics at grapevine phenological stages}

Significant variances in the fungal diversity were recorded at each grapevine habitat over time (Table 1, Fig. 2A). Soil fungi showed higher diversity than that of grapevine organs (except flowers) throughout the growing season, with a rapid increase before fruit set, and then a decrease by veraison, and finally an increase at harvest. The trend was also observed in root fungal diversity. After fruit set, the diversity of grape fungi significantly increased (ANOVA, $\mathrm{F}_{(2,27)}=6.392, p<0.001$ ) over the development and ripening process, reaching a maximum at harvest. For leaves, the fungal communities tended to be less complex and diverse as grapevine development progressed, although an increased Shannon index was observed at veraison. Interestingly, veraison was found to be a key stage of diversity changes in grapevine-associated fungi microbiota (Fig. 2A). There were significant differences in fungal community composition over time regardless of grapevine habitat (Table 1). We observed the most distinct pattern in grapes with the highest $\mathrm{R}^{2}$ coefficients (PERMANOVA, $\mathrm{R}^{2}=0.426, p<0.001$ ), followed by the soil (PERMANOVA, $\mathrm{R}^{2}=0.229, p<$ 0.001). As an example, PCoA showed a strong separation of grape samples, especially samples at harvest from earlier stages on PC1, which explained 54.9\% of the variance (Fig. 2B).

\section{The core microbiome fluctuates according to plant development}

Here, we prioritised the core microbiome over space and time to further investigate fungal community succession in the vineyard. We first identified the most dominant OTUs based on the abundance-occupancy distributions (see Experimental procedures for details) to include in the core (see Table S1 for a complete list) for the grapevine and the soil, respectively. About $1.51 \%$ of OTUs (15 OTUs) constituted a core that accounted for $74.70 \pm 2.70 \%$ of the sequences across all plant samples (Fig. 3A; Table S1A). On average, the core community was highly abundant in each grapevine habitat, with a maximum recorded in grapes (90.55 \pm $2.27 \%$ in relative abundance) (Fig. 3B; Table S1A). A wide variety of fungi was observed within those core members, and included fermentative yeasts (Saccharomyces, Debaryomyces), yeast-like fungi (Aureobasidium, Cryptococcus, Vishniacozyma), filamentous fungi (Cladosporium, Alternaria, Penicillium, 
Fusarium), and other genera (Mycosphaerella, Didymella, Ramularia, Epicoccum) (Table S1A). Within grapevine habitats, some species outside the grapevine core dominated and developed over time and also defined the core specific to this organ (Table S1A1 - A4). Specifically, three core species from Mortierella genus and Microascaceae family were as part of core community of flowers (Table S1A1), with three from Filobasidium and Alternaria genera for grapes (Table S1A2), and three from Hypocreales order, Thermoascaceae family and Lophiostoma genus for roots (Table S1A4). For leaves, only eight OTUs within the grapevine core dominated the community, while other core OTUs showed lower occupancy and/or abundance during the growth cycle (Table S1A3). For soil, 1.74\% of OTUs (35 OTUs) were the core members accounting for $63.40 \pm 3.54 \%$ of the reads across all samples, which presented a high abundance at each stage (Table S1B). The soil core taxa belonged to Ascomycota, Mortierellomycota (Mortierella), Basidiomycota (for example Clavaria and Auricularia) and Chytridiomycota (Rhizophlyctis), including some filamentous fungi like Podospora, Humicola, Ilyonectria and Sarocladium, and yeasts including Exophiala and basidiomycetous Solicoccozyma.

Tracking core OTUs within individual habitats revealed distinct temporal dynamics and succession associated with plant development (Fig. 3C - F). All the core genera in the alluvial diagrams presented significantly different relative abundances during the annual growth cycle (ANOVA, false discovery rate [FDR]-corrected $p$ value of $<0.05)$. Overall, in each habitat, veraison appeared to be a key stage where the core community was distinct from other stages (Fig. 3C - F). Some taxa changed in a similar manner in plant organs. For instance, the most dominant genera, Cladosporium, accumulated in relative abundance, between post-veraison grapes and leaves, while Aureobasidium and Cryptococcus declined in those post-veraison organs (Fig. 3C E). Fermentative yeasts S. cerevisiae and Debaryomyces hansenii persisted in the grapevine throughout the growth cycle but decreased in relative abundances of post-veraison grapevine habitats (Supplementary Table S1). However, S. cerevisiae increased in the root microbiome post-veraison. Some species like Epicoccum sp. 
and Alternaria sp. showed changes in developing grapes and became much more abundant at harvest, but these genera only showed moderate changes in leaves and roots (Fig. 3C - E; Table S1A2 - A4). It is noteworthy that a few core members only appeared or appeared in high occupancy in specific developmental stages. The best example is OTUs assigned to Alternaria infectoria and Alternaria rosae where taxa only were detected in grape samples at veraison and accumulated afterwards (Table S1A2). For the soil habitat, the most dominant genus Mortierella decreased in the relative abundance during the plant development (especially after veraison), accompanied by an increase in ascomycetous genera (Fig. 3F). Within ascomycetous genera, those taxa shared between soil and plants accumulated as the growth cycle processed, including Epicoccum, Alternaria, Fusarium, Cladosporium and Penicillium, with notably great changes at veraison, as well as soil yeasts E. equina and Solicoccozyma phenolica, and some soil-specific fungi, such as Trichoglossum, Podospora and Humicola (Fig. 3F; Table S1B).

\section{Core microbiome drives seasonal community succession}

To further confirm the robustness of these observations across the growth cycle, Random Forest supervised learning models (Breiman, 2001) were employed to classify samples and identify which taxa explain the strongest variations during grapevine developmental stages. The fungal microbiota had high discriminative power to distinguish samples coming from various stages, especially for grapes and soils (class errors, 0.100 and 0.125 on average, respectively; Table S2). Compared to all other stages, veraison had the lowest predictive error (class error $=0.150$ on average), for example, all soil samples at veraison were correctly identified (class error $=0.000$ ), suggesting that veraison was the most distinct stage of the development.

All the core OTUs were important features of these models for grapevine habitats (Fig. 4). Filamentous fungi (for example Cladosporium ramotenellum and Fusarium denticulatum), fermentative yeasts S. cerevisiae and D. hansenii, and some yeast-like fungi (Aureobasidium pullulans and Cryptococcus saitoi) within the core (Table S1) were top features in the classification models for vine habitats. Several non-core taxa were also 
important for the models (see Table S3 for a complete list). For instance, yeast Hanseniaspora uvarum (OTU_33) which was present in low occupancy $(\leq 50 \%)$, was an important feature distinguishing developmental stage, with higher abundance at veraison in grapes or harvest in leaves and roots; also, yeasts Torulaspora delbrueckii (OTU_121) were important for both grapes and leaves, and Rhodotorula babjevae (OTU_40) for grapes. In addition, some soil core species were important for random forest models of plant organs, including Mortierella spp., Sarocladium bactrocephalum, E. equina, which were present only at specific stages (Table S2B). A portion of soil core taxa explained the highest variations of some phenological stages, for instance, Epicoccum nigrum (OTU_15) and Pyrenochaetopsis leptospora (OTU_62) were the most important predictors (Fig.4; Table S1). Non-core taxa were mainly detected at early stages (flowering and fruit set; e.g., Archaeorhizomyces sp., Solicoccozyma terricola) or later stages (e.g., Penicillium multicolor, Aspergillus ardalensis), and contributed to the models. When we used the core subset to build the random forest model, a higher classification accuracy (class error $=0.075$ on average) was achieved, with improved discrimination for fruit set and harvest ( 0.200 and 0.000 , respectively). Taken together, these results confirmed that plant development stages can be distinguished based on the associated core microbiome and defined predictive features for dynamic fungal ecology in the vineyard.

\section{Co-occurrence patterns between the core microbiome and vineyard weather}

During the annual growth cycle, all weather parameters displayed significant differences between the phenological stages (Supplementary Table S4). Stage-dependent patterns in grapevine-associated microbiota (Figure 3; Supplementary Table S1, S3) also suggest that longitudinal environmental conditions are likely responsible for structuring the microbial communities. Network analysis was conducted to explore the cooccurrence patterns between plant core microbiome and environmental matrices including abiotic (weather) and biotic factor (soil microbiota), based on the strong correlation coefficients (Spearman correlation coefficient, $\rho \geq 0.8 ; \mathrm{p}<0.01$ ) (Fig. 5). Overall, the plant microbiota presented high connectivity with soil microbiota and weather conditions. The more densely connected module was observed in grape microbiota 
(average degree $=3.610$; Fig. 5A) than leaf (average degree $=2.381$; Fig. 5B) and root (average degree $=$ 2.316; Fig. 5C). In addition, grape microbiota was significantly correlated with each weather parameter. Across habitats, the most closely connected fungi were A. pullulans (OTU_1), C. ramotenellum (OTU_2), C. saitoi (OTU_3), and F. denticulatum (OTU_2019). Correspondingly, high-connectivity nodes with these OTUs were soil species Penicillium sp., Auricularia sp., Humicola sp., Mortierella sp., and Ilyonectria sp., and changing solar radiation (Env1) and vine water status related parameters (Env6, evaporation; Env8, relative soil moisture). Likewise, soil taxa were also strongly correlated with weather parameters (in particular Env1, 6, 8) (Supplementary Fig. S1). In addition, habitat-specific correlations were also found. For example, grape-specific core taxa A. infectoria (OTU_74) and root-specific core taxa Lophiostoma sp. (OTU_22) were densely connected nodes for them, respectively. The below ground root microbiota were significantly correlated with relative soil moisture.

\section{Discussion}

Microbiomes are dynamic over space and time. Despite the overwhelming diversity of microbial communities, relatively few microbial taxa dominate the ecosystems locally or globally (Delgado-Baquerizo et al., 2018; Egidi et al., 2019; Grady et al., 2019; Shade and Stopnisek, 2019). Describing the core microbiome is essential for unravelling the stable, consistent components across complex microbial assemblages. The definition of a core microbiome provides the scope to define a reproducible and conservative perspective for the dynamic microbiome, and allows for systematic discovery of persistent taxa, especially for microbiota associated with perennial plants (Shade and Stopnisek, 2019). Here, our study narrows down the immense number of measurable fungal taxa to the core microbiome to advance understanding of grapevine-associated fungal microbiota. This is an important step to further investigate contributions of fungi to functioning of the vineyard ecosystem and opens the potential to use specific fungi to deliberately manipulate the microbiome to affect grapevine health, growth, and grape and wine production. 


\section{Community succession of the grapevine-associated microbiota over space and time}

Our data show that fungal communities are not stochastically assembled but have a distinct community with habitat patterns according to the development stage of grapevines (Fig. 1). Fungal communities varying by sites have previously been shown in vineyard systems at small scales (Coller et al., 2019; Knight et al., 2019). In our study, grapevine habitats and plant development within the sampled vineyards outweighed biogeographic trends at this scale $(5 \mathrm{~km})$, which were only significant for root fungal diversity, suggesting that fungal assemblages have extensive local heterogeneity associated with their host plant (Table 1). Grapevines maintain distinct fungal microbial communities in a habitat-specific manner, but also share many taxa. Fungal diversity is higher below the ground (root zone soil, roots) than the aboveground habitats (flowers, leaves, and grapes), and this is in line with other studies on grapevines (Zarraonaindia et al., 2015; Morrison-Whittle and Goddard, 2017; Deyett and Rolshausen, 2020). Soil has been suggested as the source and reservoir of plant microbiota (Zarraonaindia et al., 2015; Morrison-Whittle and Goddard, 2018; Grady et al., 2019) yet many core taxa associated with grapevine organs (for example, Cladosporium, Aureobasidium, and Saccharomyces) are in low abundances in our soil samples (Fig. 1B, 1D). This differentiation indicates that there are ecological filters operated by the plant to favour some species while excluding others (Chesson, 2000; Leibold and McPeek, 2006). The core taxa found here persist across habitats in differing abundance with some species only existing to a specific plant organ. We observed a decrease in fungal diversity between the root zone soil and the roots but an enrichment of saprophytic fungi (Mycosphaerella and Lophiostoma, core taxa in roots; Fig. 3E) and dilution of potentially pathogenic fungi (Ilyonectria, core taxa in soil; Fig. 3F). These fungi have been previously reported colonising the rhizocompartments of grapevines (del Pilar Martínez-Diz et al., 2019; Deyett and Rolshausen, 2020). Arbuscular mycorrhizal fungi including Glomeromycota: Rhizophagus, Funneliformis, and Acaulospora, which positively affect grapevine growth in a mutualistic manner (White, 2015), were recovered with low prevalence and abundance in root zone soil and root samples in our study (data not shown). This could be due in part to the choice of barcoding region used here, rather than the large 
subunit rRNA, which is the preferred biomarker for these fungi (Lekberg et al., 2012). However, geographic location may also influence distribution (Kivlin et al., 2011; Hazard et al., 2013), as found in vineyards by Coller et al. (2019) who retrieved Glomeromycota as a core phylum in Italy, using the same ITS1F/2 primers as in our study. Interestingly, the genus Mortierella (Mortierellomycota phylum), an important component of phosphorus cycling in the rhizosphere (Osorio and Habte, 2001), were observed as core members associated with both soil and flowers (Fig. 1B; Supplementary Table S1). The presence of this genus in flowers is unexpected and may point to a role of flowers in forming and establishing the grapevine microbiome and their functionality.

The grapevine-associated microbiota are also affected by the plant development stage. The leaf fungal diversity decreased during the growth cycle (Fig. 2A), as previously described for grapevine and other plant systems (Pinto et al., 2014; Copeland et al., 2015). This may be partly attributed to experiencing greater climatic stress (UV radiation, temperature, and humidity) in the phyllosphere and thus only eight core OTUs persist over time due to environmental fluctuations. Microbial diversity increased in the grapes from fruit set to harvest stage (Fig. 2A) and may be explained by sugar accumulation and exudation as ripening proceeds. It is well documented that accumulation of sugars favours fungal colonisation (Renouf et al., 2005; Martins et al., 2014). Similar trends between soil and root fungal diversity were as expected, suggesting that root microbiomes are partly derived from the rhizosphere (Compant et al., 2011; Lundberg et al., 2012; Deyett and Rolshausen, 2020), and in turn, that root morphology and root exudation can influence the composition of the rhizosphere microbiome (Berg and Smalla, 2009; Chaparro et al., 2014). During the growing season, vineyards were treated regularly with sulphur- and copper-based fungicides. The influences of fungicides on microbial diversity and composition associated grapevine have been widely discussed (Setati et al., 2012; Martins et al., 2014; Morrison-Whittle and Goddard, 2017). Whilst this is non-negligible, the fact of microbial assemblage and community succession according to plant development is of significance. The dominance of 
yeast-like fungus $A$. pullulans in the vineyard has previously been attributed to its resistance to fungicides, that the ability to detoxify copper compete against other fungi (Comitini and Ciani, 2008; Schmid et al., 2011). Martins et al. (2014) demonstrated that growth-stage factor was preponderant in shaping fungal diversity and richness in grape surface throughout the ripening process, followed by fungicide treatments (encapsulated in the farming system).

Our study shows that a core microbiome of grapevines exists over space and time in the vineyard ecosystem. Core microorganisms are expected to play important roles in organising assembly of plant-associated microbiomes rather than promoting plant growth directly (Toju et al., 2018). A core microorganism can be a mutualist, a commensalist or even an antagonist when it is the sole inoculum in a plant (Lundberg et al., 2012; Toju et al., 2018). In our study, the core species Mycosphaerella tassiana, Cladosporium ramontellum, Didymella negriana are normally considered to be grapevine pathogens, whereas we observed no symptoms of plant diseases in the vines. We cannot easily explain these results but suggest that the induction of disease by these species may be dependent not just on presence, but the community-scale influences or other plant factors. We observed an increase in the relative abundance of the soil core taxa that were shared with plants over the growing cycle, and this lends further support to the influence from the roots and plant development on the soil microbiome (Fig. 3F). Aureobasidium are the most widespread fungi in the vineyard and persist throughout the growing season and decline at later stages across plant organs (Fig. 3), supporting previous studies in vineyard ecosystems (Renouf et al., 2005; Martins et al., 2014; Pinto et al., 2014). Using highthroughput sequencing, our data reveal the persistence of $S$. cerevisiae across habitats and developmental stages as core taxa, which has previously been detected in mature grapes based on enrichment culturedependent methods (Fleet et al., 2002; Mannazzu et al., 2002; Renouf et al., 2005) and at low frequencies in leaves (Pinto et al., 2014). We did not observe an expected increase in the relative abundance of S. cerevisiae after veraison in grape and leaf samples (Fig. 3C, 3D), but there could be increased competition with other 
species, for example Cladosporium as sugar becomes more available. Meanwhile, some non-core OTUs displayed temporal succession, which were screened by a supervised learning approach (Fig. 4). These predictors associated with grapevine displayed seasonal volatility, for instance, Filobasidium stepposum, E. equina, and Clonostachys rosea were not detected at veraison yet reappeared at harvest in roots, as did $M$. amoeboidea for leaves, and S. bactrocephalum for grapes (Fig.4; Supplementary Table S3). Cyclic patterns have been reported in the human gut microbiome and indicate that some dynamic lineages of microbes decreased in prevalence and abundance in modernised populations (Smits et al., 2017). Conditionally rare taxa (here, specific to the habitat and the development stage) can disproportionately contribute to temporal changes in microbial communities, for example, acting as a reservoir that can rapidly respond to environmental changes, thus playing roles in ecosystem resilience and stability (Shade et al., 2014; Lynch and Neufeld, 2015). In this study, those non-core OTUs were not considered as the main driver of seasonal community succession due to lack of persistence and clear relationships with grapevines and wine fermentation, but their dynamics provide a more complete view of microbial diversity and also indicate some adaptation process for the plant or plant-microbe interactions. Further research will determine if rare taxa and related volatility have an ecological or functional role in the vineyard.

\section{Grapevine-associated microbiome in response to plant development and the changing}

\section{environment}

The microbiome succession is associated with plant development. Studies have shown that phyllosphere (Copeland et al., 2015) and rhizospheric fungal and bacterial communities of a wide range of plants, such as Arabidopsis, Medicago, wheat, maize, pea, and sugar beet, change according to plant developmental stages (Baudoin et al., 2002; Mougel et al., 2006; Houlden et al., 2008; Micallef et al., 2009). In our study, veraison is the stage where the fungi microbiome of the grapevine changes very distinctly. Significant changes in the fungal diversity, and most distinct community structures of both the core (Fig. 3) and the whole (Supplementary Table S2) datasets were observed at veraison. In particular, this observation is clearer in root 
zone soil and grape microbiome, in which many non-core OTUs were only detected before or after veraison (Supplementary Table S3). In grapevine phenology, veraison marks the beginning of ripening and is a crucial time point of grapevine metabolism and growth. Besides colour change in the berries due to anthocyanin production and accumulation, berries soften as pectin and cellulose are degraded, acidity declines, and sugar accumulates. All these factors create a more favourable environment for microbial colonisation in grapes (Coombe, 1992; Renouf et al., 2005). Mougel et al. (2006) recorded that for Medicago truncatula the rhizosphere microbial communities differed according to the plant development stage, with the most significant shifts observed during the transition from vegetative to reproductive stages. Mounting evidence suggest regulation by root exudates as one mechanism (Broeckling et al., 2008; Chaparro et al., 2013; Chaparro et al., 2014; Zhalnina et al., 2018). Plants secrete blends of compounds and specific phytochemicals (sugars, sugar alcohols, phenolics, and amino acids) in the root exudates that are differentially produced at distinct developmental stages acting as signalling molecules to help orchestrate rhizosphere microbiome assemblage (Chaparro et al., 2014). Grapevines may select a subset of microbes at different stages of development presumably for specific functions that the core microbiome or the rare taxa express and thus improving plant fitness in the vineyard ecosystem. Future experiments targeting rhizosphere microbiome and chemical environments are essential to elucidate the exact mechanism.

Alongside the influences of changing metabolism and physiology during plant development, grapevineassociated microbiome is also subject to dynamic environmental conditions where plants grow. Soil core microbiome closely correlated with the abiotic matrices (weather parameters; Supplementary Fig. S1), and it is in turn the biotic factor for the plant microbiome. Different plant organs respond to changes in their environment in different manners, via different and/or habitat-specific core taxa closely correlated with different environmental matrices (Fig. 5). Some important features distinguishing stages of soil microbiome identified by random forest models are also highly connected with plant core microbiome, including 
Mortierella sp., Penicillium sp., and Ilyonectria sp.. Water status (evaporation and relative soil moisture) and solar radiation had a strong correlation with the microbiome. For instance, higher solar radiation and temperature, decreased precipitation, increased evaporation and transpiration, and lower relative soil moisture were observed in the period from fruit set to veraison in both vineyards (data not shown), and this water stress event coincided with dramatic changes in the microbiome during the period discussed above. Meanwhile, drought triggers substantial plant responses, from physiological changes including root-shoot signaling (for example abscisic acid), the decreased photosynthesis and chlorophyll contents, disturbed plant water relations and osmolyte accumulation, to metabolic perturbations like nitrogen metabolism and secondary metabolite synthesis (Anjum et al., 2011; Hochberg et al., 2013), which may also affect grapevine-associated fungal communities. In viticulture, moderate water stress during ripening is recognised as beneficial for grape quality, resulting in lower yields and a concentration of berry metabolites (Van Leeuwen and Seguin, 2006). Our work may provide a microbial insight to understand additional mechanisms of how water status can affect quality wine production.

Global studies show that climate (especially precipitation) (Tedersoo et al., 2014; Cavicchioli et al., 2019), followed by edaphic factors (such as soil pH, aridity) (Maestre et al., 2015; Bahram et al., 2018), drive soil fungal community assembly and hence multifunctionality in ecosystems (Bahram et al., 2018; Cavicchioli et al., 2019; Li et al., 2019). Our recent study revealed that wine-related fungal communities structured and distinguished vineyard ecosystems by impacting the flavour and quality of wine, and weather (in particular solar radiation and temperature) strongly affected soil and must fungal communities and the resultant wines across six winegrowing regions in southern Australia (Liu et al, under review). Vine water availability can condition the grape and must microbiota at regional scales, for example, precipitation and humidity correlates with the presence of filamentous fungi (for example Penicillium) (Bokulich et al., 2014) and yeasts (such as Saccharomyces, Hanseniaspora, and Metschnikowia) (Jara et al., 2016). In the present study, microbial 
heterogeneity and seasonal dynamics across habitats highlight that water availability may influence the microbiota present within vineyards. Grapevine phenology is shifting with warming and drying induced by climate change (Webb et al., 2012; Cameron et al., 2020), and our work suggests that the grapevine-associated microbiota will change as well, thus potentially affect the distinctiveness of regional wines (Liu et al., under review). Microbial assemblages responding to climate change may also affect plant physiology, as reported for rhizosphere microorganisms which influence plant phenology and alter the flowering time (Wagner et al., 2014; Panke-Buisse et al., 2015; Lu et al., 2018). Interactions between grapevines and associated fungi deserve future study and would provide further perspectives on vineyard establishment and management practices such as managing soil moisture and crop yield (Webb et al., 2012; Palliotti et al., 2014). As agricultural practices adapt to a changing climate, understanding the core microbiome, transient taxa and specific interactions between plant and microbe become more important to maintaining plant productivity (Cavicchioli et al., 2019).

\section{Conclusions}

Our study describes the core microbiome of grapevines and illustrates the seasonal community succession driven by the core microbiome. Plants recruit their microorganisms based on habitats at different stages of development yet conserve core fungal species. While undertaken at two sites in one region in Australia, our research highlights the need to further investigate core microbial members for functions and interactions with the grapevine to understand the interactive functionalities that occur between the host crop and their microbiome. Increasing our understanding of fungal ecology in the vineyard and their responses to the changing environment over the course of the growing season would help improve management techniques to maintain healthy vines, produce quality grapes and allow expression of wine regionality. 


\section{Experimental procedures}

\section{Sample sites}

Grapevine samples were collected at two vineyards in Mornington Peninsula wine region during the 2018 vintage (Fig. S2; grapevines were created with BioRender, https://app.biorender.com/). Vineyard A (1.4 ha, altitude 55 m) and B (1.7 ha, altitude 195 m) are 5 km apart, planted with Vitis vinifera cv. Pinot Noir (clone MV6). Both vineyards are commercially operated by a single winery under very similar viticultural practices. Grape marc was spread at 2 tonnes per hectare for both vineyards in August 2017. Grapevines were under vertical shoot positioning trellising systems and were treated with fungicides of sulphur (Microthio Disperse), copper hydroxide (Kocide), metalaxyl + copper hydroxide (Ridomil), and myclobutanil (Mycloss) for the protection of powdery mildew and downy mildew, from shoot growth (October 2017) until pre-veraison (January 2018) (see Supplementary Table S5). In each vineyard, five replicate vines were selected from the top, middle, and bottom of the dominant slope covering topological profiles of the vineyard, with pairwise distances ranging from $15 \mathrm{~m}$ to $135 \mathrm{~m}$ (Fig. S2A). Soil types in both vineyards are clay loam (Isbell 2016). Soil chemical analyses (pH, carbon, nitrogen) did not show significant differences between vineyards at harvest (Supplementary Table S6). GPS coordinates were recorded for each site and utilised to extract weekly weather data provided by Australian Water Availability Project (AWAP) (Jones et al., 2009) from two weather stations. Variables were observed by robust topography resolving analysis methods at a resolution of $0.05^{\circ} \times 0.05^{\circ}$ (approximately $5 \mathrm{~km} \mathrm{x} 5 \mathrm{~km}$ ). Weekly measurements were extracted for precipitation (m), solar radiation $\left(\mathrm{MJ} / \mathrm{m}^{2}\right)$, total evaporation $(\mathrm{m})$ (soil + vegetation), transpiration $(\mathrm{m})$, mean high temperature $\left({ }^{\circ} \mathrm{C}\right)$, mean low temperature $\left({ }^{\circ} \mathrm{C}\right)$, mean temperature $\left({ }^{\circ} \mathrm{C}\right)$, and mean relative soil moisture for each development stage during the growing season (October 2017 - April 2018). 


\section{Sample collection}

In each grapevine, four different grapevine habitats were studied: root zone soil, roots, leaves, and grapes or flowers (season dependent) (Fig. S2B). Samples were aseptically collected from the same vines at four developmental stages [as defined by Coombe (1992)] at flowering (November 2017), fruit-set (young berries enlarging, December 2017), veraison (berry colour change, January - February 2018), and harvest (berries ripe, March 2018) from both vineyards. Root zone soil was taken from the surrounding roots at the depth of 0 - $15 \mathrm{~cm}$. Three sub-samples (the sun-exposed side, the shade side, and the middle) were mixed to form a composite sample. Healthy leaves of similar sizes, with no discernible evidence of disease were collected. Undamaged grapes were chosen from different grape bunches. Samples were immediately stored in sterile bags and transported to the laboratory on ice. Roots were collected and rinsed and cleaned of all visible soil using distilled water and flame-sterilized utensils back in the laboratory. Plant materials were flash frozen in liquid nitrogen and all the samples were stored at $-80{ }^{\circ} \mathrm{C}$ until processing. A total of 160 vineyard samples were collected and processed.

\section{DNA extraction and sequencing}

Genomic DNA was extracted from soil and botanical samples using PowerSoil" ${ }^{\mathrm{TM}}$ DNA Isolation kits (QIAgen, CA, USA). DNA isolation of soil (0.25 g) samples followed the kit protocol. For plant parts, roots, leaves, grapes (removed seeds and stems) or flowers, were ground into powder in liquid nitrogen with $1 \%$ polyvinylpolypyrrolidone and then DNA isolated following the kit protocol. Thus, endophytes and epiphytes were extracted, sequenced, and analysed simultaneously from all plant organs. DNA extracts were stored at $20{ }^{\circ} \mathrm{C}$ until further analysis.

Genomic DNA was submitted to Australian Genome Research Facility (AGRF) for amplification and sequencing. To assess the fungal communities, partial fungal internal transcribed spacer (ITS) region was amplified using the universal primer pairs ITS1F/2 (Gardes and Bruns, 1993). The primary PCR reactions 
contained 10 ng DNA template, 2× AmpliTaq Gold® 360 Master Mix (Life Technologies, Australia), 5 pmol of each primer. A secondary PCR to index the amplicons was performed with TaKaRa Taq DNA Polymerase (Clontech). Amplification was conducted under the following conditions: $95{ }^{\circ} \mathrm{C}$ for $7 \mathrm{~min}$, followed by 35 cycles of $94^{\circ} \mathrm{C}$ for $30 \mathrm{~s}, 55^{\circ} \mathrm{C}$ for $45 \mathrm{~s}, 72{ }^{\circ} \mathrm{C}$ for $60 \mathrm{~s}$ and a final extension at $72{ }^{\circ} \mathrm{C}$ for $7 \mathrm{~min}$. The resulting amplicons were cleaned again using magnetic beads, quantified by fluorometry (Promega Quantifluor) and normalised. The equimolar pool was cleaned a final time using magnetic beads to concentrate the pool and then measured using a High-Sensitivity D1000 Tape on an Agilent 2200 TapeStation. The pool was diluted to 5nM and molarity was confirmed again using a High-Sensitivity D1000 Tape.This was followed by 300 bp paired-end sequencing on an Illumina MiSeq (San Diego, CA, USA).

Raw sequences were processed using QIIME v1.9.2 (Caporaso et al., 2010). Low quality regions (Q < 20) were trimmed from the 5 ' end of the sequences, and the paired ends were joined using FLASH (Magoč and Salzberg, 2011). Primers were trimmed and a further round of quality control was conducted to discard full length duplicate sequences, short sequences ( $<100 \mathrm{nt}$ ), and sequences with ambiguous bases. Sequences were clustered followed by chimera checking using UCHIME algorithm from USEARCH v7.1.1090 (Edgar et al., 2011). Operational taxonomic units (OTUs) were assigned using UCLUST open-reference OTU-picking workflow with a threshold of 97\% pairwise identity (Edgar, 2010). Singletons or unique reads in the resultant data set were discarded. Taxonomy was assigned to OTUs in QIIME using the UNITE fungal ITS database (v7.2) (Kõljalg et al., 2005) and BLAST classifier. To avoid/reduce biases generated by varying sequencing depth, sequences were rarefied to 10,000 per sample (the lowest sequencing depth) prior to downstream analysis. Raw sequencing reads have been deposited at the National Centre for Biotechnology Information Sequence Read Archive under the bioproject PRJNA626608. 


\section{Data analysis}

Fungal OTUs shared among habitats in the vineyard was illustrated by the "VennDiagram" package (Chen and Boutros, 2011) in R (v3.5.0). Microbial alpha-diversity was calculated using the Shannon index with the “vegan” package (Oksanen et al., 2007). One-way analysis of variance (ANOVA) was used to determine whether sample classifications (e.g., habitat, developmental stage, vineyard) contained statistically significant differences in the diversity. Principal coordinate analysis (PCoA) was performed to evaluate the distribution patterns of grapevine-associated microbiome based on beta-diversity calculated by the Bray-Curtis distance with the "labdsv" package (Roberts, 2007). Permutational multivariate analysis of variance (PERMANOVA) using distance matrices with 999 permutations was conducted within each sample category to determine the statistically significant differences with “adonis” function in “vegan” (Oksanen et al., 2007). Significant taxonomic differences of fungi between vineyard habitats were tested using linear discriminant analysis (LDA) effect size (LEfSe) analysis (Segata et al., 2011) (https://huttenhower.sph.harvard.edu/galaxy/). The OTU table was filtered to include only OTUs $>0.1 \%$ relative abundance to reduce LEfSe complexity. The factorial Kruskal-Wallis sum-rank test $(\alpha=0.05)$ was applied to identify taxa with significant differential abundances between classes (all-against-all comparisons), followed by the logarithmic LDA score (threshold $=2.0$ ) to estimate the effect size of each discriminative feature. Significant taxa were used to generate taxonomic cladograms illustrating differences between sample categories.

The core microbiome was defined based on abundance-occupancy distributions that include highly abundant and ubiquitous taxa (Delgado-Baquerizo et al., 2018; Shade and Stopnisek, 2019). For soil core microbiome, OTUs were filtered out with top 1\% mean relative abundance (MRA) across all samples (Galand et al., 2009) and an occurrence frequency found in more than $50 \%$ of samples or $100 \%$ occupancy at any time point (Delgado-Baquerizo et al., 2018; Grady et al., 2019). For the grapevine, OTUs with top 10\% MRA across all samples (roots, leaves, grapes, flowers) and 50\% occupancy of all samples or $100 \%$ occupancy of one habitat samples were defined as the core members (Delgado-Baquerizo et al., 2018; Grady et al., 2019). For each 
plant organ (except flowers), the core OTUs were those top 10\% abundant across all samples with 50\% occupancy of all samples or 100\% occupancy at any developmental stage (Delgado-Baquerizo et al., 2018; Grady et al., 2019). Dynamics and successions of the core microbiome were illustrated by alluvial diagrams using the "ggalluvial” package (Brunson, 2018) in "ggplot2”.

Random forest supervised classification models (Breiman, 2001) were used to evaluate the discriminative power of fungal microbiota (all OTUs) to distinguish developmental stages and the robustness of the groupings themselves. The importance of each predictor is measured by the mean decrease in Gini coefficient (MDG) (that is, how each variable contributes to the homogeneity of the nodes and leaves in the resulting random forest, and variables resulting in nodes with higher purity have a higher MDG). Models were constructed with 10,000 trees, with OTUs as predictors and developmental stages as class labels using the “randomForest” package (Liaw and Wiener, 2002).

The co-occurrence/interaction patterns between the core microbiome associated with grapevine habitats (grapes, leaves, roots) and soil, and weather conditions during the growing season was explored using network analysis with Cytoscape (Shannon et al., 2003). Correlation matrices were calculated with all possible pairwise Spearman's rank correlations between selected OTUs of plant and soil, or OTUs and weather indexes. Correlations with a Spearman correlation coefficient $\rho \geq 0.8$ and a $p<0.01$ were considered statistically robust and displayed in the networks (Junker and Schreiber, 2008).

\section{Acknowledgements}

We give our sincere thanks to the vignerons in Mornington Peninsula who kindly allowed vineyard access and enabled sampling. DL acknowledges support from a Ph.D. scholarship and funding from Wine Australia (AGW Ph1602) and a Melbourne Research Scholarship from the University of Melbourne. Dr Qinling Chen, Prof Deli Chen and Dr Pangzhen Zhang are acknowledged for helpful discussions and support in the course of this work. 


\section{Conflict of Interest}

We declare that this research was conducted in the absence of any commercial or financial relationships that could be constructed as a potential conflict of interest.

This article is protected by copyright. All rights reserved. 


\section{References}

Anjum, S.A., Xie, X.-y., Wang, L.-c., Saleem, M.F., Man, C., and Lei, W. (2011) Morphological, physiological and biochemical responses of plants to drought stress. African journal of agricultural research 6: 2026-2032.

Bahram, M., Hildebrand, F., Forslund, S.K., Anderson, J.L., Soudzilovskaia, N.A., Bodegom, P.M. et al. (2018) Structure and function of the global topsoil microbiome. Nature 560: 233-237.

Barata, A., Malfeito-Ferreira, M., and Loureiro, V. (2012) The microbial ecology of wine grape berries. International journal of food microbiology 153: 243-259.

Baudoin, E., Benizri, E., and Guckert, A. (2002) Impact of growth stage on the bacterial community structure along maize roots, as determined by metabolic and genetic fingerprinting. Applied Soil Ecology 19: 135-145. Berg, G., and Smalla, K. (2009) Plant species and soil type cooperatively shape the structure and function of microbial communities in the rhizosphere. FEMS microbiology ecology 68: 1-13.

Bokulich, N.A., Thorngate, J.H., Richardson, P.M., and Mills, D.A. (2014) Microbial biogeography of wine grapes is conditioned by cultivar, vintage, and climate. Proceedings of the National Academy of Sciences 111: E139-E148.

Breiman, L. (2001) Random forests. Machine learning 45: 5-32.

Broeckling, C.D., Broz, A.K., Bergelson, J., Manter, D.K., and Vivanco, J.M. (2008) Root exudates regulate soil fungal community composition and diversity. Appl Environ Microbiol 74: 738-744.

Brunson, J.C. (2018) Ggalluvial: Alluvial diagrams in'ggplot2'. R package version 0.9. 1. In.

Burns, K.N., Kluepfel, D.A., Strauss, S.L., Bokulich, N.A., Cantu, D., and Steenwerth, K.L. (2015) Vineyard soil bacterial diversity and composition revealed by $16 \mathrm{~S}$ rRNA genes: Differentiation by geographic features. Soil Biology and Biochemistry 91: 232-247.

Cameron, W., Petrie, P.R., Barlow, E.W.R., Patrick, C.J., Howell, K., and Fuentes, S. (2020) Advancement of grape maturity: comparison between contrasting cultivars and regions. Australian Journal of Grape and Wine Research 26: 53-67.

Caporaso, J.G., Kuczynski, J., Stombaugh, J., Bittinger, K., Bushman, F.D., Costello, E.K. et al. (2010) QIIME allows analysis of high-throughput community sequencing data. Nature methods 7: 335.

Cavicchioli, R., Ripple, W.J., Timmis, K.N., Azam, F., Bakken, L.R., Baylis, M. et al. (2019) Scientists' warning to humanity: microorganisms and climate change. Nature Reviews Microbiology.

Chaparro, J.M., Badri, D.V., and Vivanco, J.M. (2014) Rhizosphere microbiome assemblage is affected by plant development. The ISME journal 8: 790.

Chaparro, J.M., Badri, D.V., Bakker, M.G., Sugiyama, A., Manter, D.K., and Vivanco, J.M. (2013) Root exudation of phytochemicals in Arabidopsis follows specific patterns that are developmentally programmed and correlate with soil microbial functions. PloS one $\mathbf{8}$.

Chen, H., and Boutros, P.C. (2011) VennDiagram: a package for the generation of highly-customizable Venn and Euler diagrams in R. BMC bioinformatics 12: 35.

Chesson, P. (2000) Mechanisms of maintenance of species diversity. Annual review of Ecology and Systematics 31: 343-366.

Ciani, M., Comitini, F., Mannazzu, I., and Domizio, P. (2010) Controlled mixed culture fermentation: a new perspective on the use of non-Saccharomyces yeasts in winemaking. FEMS yeast research 10: 123-133.

Coller, E., Cestaro, A., Zanzotti, R., Bertoldi, D., Pindo, M., Larger, S. et al. (2019) Microbiome of vineyard soils is shaped by geography and management. Microbiome 7: 140.

Comitini, F., and Ciani, M. (2008) Influence of fungicide treatments on the occurrence of yeast flora associated with wine grapes. Annals of microbiology 58: 489-493.

Compant, S., Mitter, B., Colli-Mull, J.G., Gangl, H., and Sessitsch, A. (2011) Endophytes of grapevine flowers, berries, and seeds: identification of cultivable bacteria, comparison with other plant parts, and visualization of niches of colonization. Microbial ecology 62: 188-197. 
Coombe, B. (1992) Research on development and ripening of the grape berry. American Journal of Enology and Viticulture 43: 101-110.

Copeland, J.K., Yuan, L., Layeghifard, M., Wang, P.W., and Guttman, D.S. (2015) Seasonal community succession of the phyllosphere microbiome. Molecular Plant-Microbe Interactions 28: 274-285.

Delgado-Baquerizo, M., Oliverio, A.M., Brewer, T.E., Benavent-González, A., Eldridge, D.J., Bardgett, R.D. et al. (2018) A global atlas of the dominant bacteria found in soil. Science 359: 320-325.

Deyett, E., and Rolshausen, P.E. (2020) Endophytic Microbial Assemblage in Grapevine. FEMS Microbiology Ecology.

Edgar, R.C. (2010) Search and clustering orders of magnitude faster than BLAST. Bioinformatics 26: 24602461.

Edgar, R.C., Haas, B.J., Clemente, J.C., Quince, C., and Knight, R. (2011) UCHIME improves sensitivity and speed of chimera detection. Bioinformatics 27: 2194-2200.

Egidi, E., Delgado-Baquerizo, M., Plett, J.M., Wang, J., Eldridge, D.J., Bardgett, R.D. et al. (2019) A few Ascomycota taxa dominate soil fungal communities worldwide. Nature communications 10: 1-9.

Fierer, N., and Jackson, R.B. (2006) The diversity and biogeography of soil bacterial communities.

Proceedings of the National Academy of Sciences of the United States of America 103: 626-631.

Fleet, G., Prakitchaiwattana, C., Beh, A., and Heard, G. (2002) The yeast ecology of wine grapes. Biodiversity and biotechnology of wine yeasts Research Signpost, Kerala, India 95: 1-17.

Galand, P.E., Casamayor, E.O., Kirchman, D.L., and Lovejoy, C. (2009) Ecology of the rare microbial biosphere of the Arctic Ocean. Proceedings of the National Academy of Sciences 106: 22427-22432.

Gardes, M., and Bruns, T.D. (1993) ITS primers with enhanced specificity for basidiomycetes-application to the identification of mycorrhizae and rusts. Molecular ecology 2: 113-118.

Gayevskiy, V., and Goddard, M.R. (2012) Geographic delineations of yeast communities and populations associated with vines and wines in New Zealand. The ISME journal 6: 1281-1290.

Gilbert, J.A., van der Lelie, D., and Zarraonaindia, I. (2014) Microbial terroir for wine grapes. Proceedings of the National Academy of Sciences 111: 5-6.

Goddard, M.R., Anfang, N., Tang, R., Gardner, R.C., and Jun, C. (2010) A distinct population of

Saccharomyces cerevisiae in New Zealand: evidence for local dispersal by insects and human-aided global dispersal in oak barrels. Environmental microbiology 12: 63-73.

Grady, K.L., Sorensen, J.W., Stopnisek, N., Guittar, J., and Shade, A. (2019) Assembly and seasonality of core phyllosphere microbiota on perennial biofuel crops. bioRxiv: 446369.

Hazard, C., Gosling, P., Van Der Gast, C.J., Mitchell, D.T., Doohan, F.M., and Bending, G.D. (2013) The role of local environment and geographical distance in determining community composition of arbuscular mycorrhizal fungi at the landscape scale. The ISME journal 7: 498-508.

Hochberg, U., Degu, A., Toubiana, D., Gendler, T., Nikoloski, Z., Rachmilevitch, S., and Fait, A. (2013) Metabolite profiling and network analysis reveal coordinated changes in grapevine water stress response. BMC plant biology 13: 184.

Houlden, A., Timms-Wilson, T.M., Day, M.J., and Bailey, M.J. (2008) Influence of plant developmental stage on microbial community structure and activity in the rhizosphere of three field crops. FEMS Microbiology Ecology 65: 193-201.

Jara, C., Laurie, V.F., Mas, A., and Romero, J. (2016) Microbial Terroir in Chilean Valleys: Diversity of Nonconventional Yeast. Frontiers in microbiology 7.

Jumpponen, A., and Jones, K. (2010) Seasonally dynamic fungal communities in the Quercus macrocarpa phyllosphere differ between urban and nonurban environments. New Phytologist 186: 496-513. Junker, B.H., and Schreiber, F. (2008) Analysis of biological networks: Wiley Online Library. Kivlin, S.N., Hawkes, C.V., and Treseder, K.K. (2011) Global diversity and distribution of arbuscular mycorrhizal fungi. Soil Biology and Biochemistry 43: 2294-2303. 
Knight, S., and Goddard, M. (2015) Quantifying separation and similarity in a Saccharomyces cerevisiae metapopulation. The ISME journal 9: 361-370.

Knight, S.J., Karon, O., and Goddard, M.R. (2019) Small scale fungal community differentiation in a vineyard system. Food Microbiology: 103358.

Kõljalg, U., Larsson, K.H., Abarenkov, K., Nilsson, R.H., Alexander, I.J., Eberhardt, U. et al. (2005) UNITE: a database providing web-based $m$ ethods for the molecular identification of ectomycorrhizal fungi. New Phytologist 166: 1063-1068.

Lam, S.S., and Howell, K.S. (2015) Drosophila-associated yeast species in vineyard ecosystems. FEMS microbiology letters 362.

Leibold, M.A., and McPeek, M.A. (2006) Coexistence of the niche and neutral perspectives in community ecology. Ecology 87: 1399-1410.

Lekberg, Y., Schnoor, T., Kjøller, R., Gibbons, S.M., Hansen, L.H., Al-Soud, W .A. et al. (2012) 454sequencing reveals stochastic local reassembly and high disturbance tolerance within arbuscular mycorrhizal fungal communities. Journal of Ecology 100: 151-160.

Li, J., Delgado-Baquerizo, M., Wang, J.-T., Hu, H.-W., Cai, Z.-J., Zhu, Y.-N., and Singh, B.K. (2019) Fungal richness contributes to multifunctionality in boreal forest soil. Soil Biology and Biochemistry 136: 107526.

Liaw, A., and Wiener, M. (2002) Classification and regression by randomForest. $R$ news 2: 18-22.

Liu, D., Zhang, P., Chen, D., and Howell, K.S. (2019) From the vineyard to the winery: how microbial ecology drives regional distinctiveness of wine. Frontiers in Microbiology 10: 2679.

Lu, T., Ke, M., Lavoie, M., Jin, Y., Fan, X., Zhang, Z. et al. (2018) Rhizosphere microorganisms can influence the timing of plant flowering. Microbiome 6: 231.

Lundberg, D.S., Lebeis, S.L., Paredes, S.H., Yourstone, S., Gehring, J., Malfatti, S. et al. (2012) Defining the core Arabidopsis thaliana root microbiome. Nature 488: 86.

Lynch, M.D., and Neufeld, J.D. (2015) Ecology and exploration of the rare biosphere. Nature Reviews Microbiology 13: 217-229.

Maestre, F.T., Delgado-Baquerizo, M., Jeffries, T.C., Eldridge, D.J., Ochoa, V., Gozalo, B. et al. (2015) Increasing aridity reduces soil microbial diversity and abundance in global drylands. Proceedings of the National Academy of Sciences 112: 15684-15689.

Magoč, T., and Salzberg, S.L. (2011) FLASH: fast length adjustment of short reads to improve genome assemblies. Bioinformatics 27: 2957-2963.

Mannazzu, I., Clementi, F., and Ciani, M. (2002) Strategies and criteria for the isolation and selection of autochthonous starters. Biodiversity and biotechnology of wine yeasts: 19-34.

Marasco, R., Rolli, E., Fusi, M., Michoud, G., and Daffonchio, D. (2018) Grapevine rootstocks shape underground bacterial microbiome and networking but not potential functionality. Microbiome 6: 3 . Martins, G., Vallance, J., Mercier, A., Albertin, W., Stamatopoulos, P., Rey, P. et al. (2014) Influence of the farming system on the epiphytic yeasts and yeast-like fungi colonizing grape berries during the ripening process. International journal of food microbiology 177: 21-28.

Martiny, J.B.H., Bohannan, B.J., Brown, J.H., Colwell, R.K., Fuhrman, J.A., Green, J.L. et al. (2006) Microbial biogeography: putting microorganisms on the map. Nature Reviews Microbiology 4: 102.

Micallef, S.A., Channer, S., Shiaris, M.P., and Colón-Carmona, A. (2009) Plant age and genotype impact the progression of bacterial community succession in the Arabidopsis rhizosphere. Plant signaling \& behavior 4: 777-780.

Morrison-Whittle, P., and Goddard, M. (2017) Fungal communities are differentially affected by conventional and biodynamic agricultural management approaches in vineyard ecosystems. Agriculture, Ecosystems and Environment.

Morrison-Whittle, P., and Goddard, M.R. (2018) From vineyard to winery: a source map of microbial diversity driving wine fermentation. Environmental microbiology 20: 75-84. 
Mougel, C., Offre, P., Ranjard, L., Corberand, T., Gamalero, E., Robin, C., and Lemanceau, P. (2006) Dynamic of the genetic structure of bacterial and fungal communities at different developmental stages of Medicago truncatula Gaertn. cv. Jemalong line J5. New Phytologist 170: 165-175.

Müller, D.B., Vogel, C., Bai, Y., and Vorholt, J.A. (2016) The plant microbiota: systems-level insights and perspectives. Annual review of genetics 50: 211-234.

Oksanen, J., Kindt, R., Legendre, P., O’Hara, B., Stevens, M.H.H., Oksanen, M.J., and Suggests, M. (2007) The vegan package. Community ecology package 10: 631-637.

Osorio, N., and Habte, M. (2001) Synergistic influence of an arbuscular mycorrhizal fungus and a P solubilizing fungus on growth and P uptake of Leucaena leucocephala in an Oxisol. Arid Land Research and Management 15: 263-274.

Palliotti, A., Tombesi, S., Silvestroni, O., Lanari, V., Gatti, M., and Poni, S. (2014) Changes in vineyard establishment and canopy management urged by earlier climate-related grape ripening: A review. Scientia Horticulturae 178: 43-54.

Panke-Buisse, K., Poole, A.C., Goodrich, J.K., Ley, R.E., and Kao-Kniffin, J. (2015) Selection on soil microbiomes reveals reproducible impacts on plant function. The ISME journal 9: 980.

Pinto, C., Pinho, D., Sousa, S., Pinheiro, M., Egas, C., and Gomes, A.C. (2014) Unravelling the diversity of grapevine microbiome. PLoS One 9: e85622.

Portillo, M.d.C., Franquès, J., Araque, I., Reguant, C., and Bordons, A. (2016) Bacterial diversity of Grenache and Carignan grape surface from different vineyards at Priorat wine region (Catalonia, Spain). International journal of food microbiology 219: 56-63.

Renouf, V., Claisse, O., and Lonvaud-Funel, A. (2005) Understanding the microbial ecosystem on the grape berry surface through numeration and identification of yeast and bacteria. Australian Journal of Grape and Wine Research 11: 316-327.

Roberts, D.W. (2007) labdsv: Ordination and multivariate analysis for ecology. R package version 1. Segata, N., Izard, J., Waldron, L., Gevers, D., Miropolsky, L., Garrett, W.S., and Huttenhower, C. (2011) Metagenomic biomarker discovery and explanation. Genome biology 12: R60.

Schmid, F., Moser, G., Müller, H., and Berg, G. (2011) Functional and structural microbial diversity in organic and conventional viticulture: organic farming benefits natural biocontrol agents. Applied and environmental microbiology 77: 2188-2191.

Setati, M.E., Jacobson, D., Andong, U.-C., and Bauer, F. (2012) The vineyard yeast microbiome, a mixed model microbial map. PLoS One 7: e52609.

Shade, A., and Gilbert, J.A. (2015) Temporal patterns of rarity provide a more complete view of microbial diversity. Trends in microbiology 23: 335-340.

Shade, A., and Stopnisek, N. (2019) Abundance-occupancy distributions to prioritize plant core microbiome membership. Current opinion in microbiology 49: 50-58.

Shade, A., Jones, S.E., Caporaso, J.G., Handelsman, J., Knight, R., Fierer, N., and Gilbert, J.A. (2014) Conditionally rare taxa disproportionately contribute to temporal changes in microbial diversity. MBio 5: e01371-01314.

Shannon, P., Markiel, A., Ozier, O., Baliga, N.S., Wang, J.T., Ramage, D. et al. (2003) Cytoscape: a software environment for integrated models of biomolecular interaction networks. Genome research 13: 2498-2504.

Smits, S.A., Leach, J., Sonnenburg, E.D., Gonzalez, C.G., Lichtman, J.S., Reid, G. et al. (2017) Seasonal cycling in the gut microbiome of the Hadza hunter-gatherers of Tanzania. Science 357: 802-806.

Stefanini, I., and Cavalieri, D. (2018) Metagenomic Approaches to Investigate the Contribution of the Vineyard Environment to the Quality of Wine Fermentation: Potentials and Difficulties. Frontiers in Microbiology 9.

Stefanini, I., Dapporto, L., Legras, J.-L., Calabretta, A., Di Paola, M., De Filippo, C. et al. (2012) Role of social wasps in Saccharomyces cerevisiae ecology and evolution. Proceedings of the National Academy of Sciences 109: 13398-13403. 
Swiegers, J., Bartowsky, E., Henschke, P., and Pretorius, I. (2005) Yeast and bacterial modulation of wine aroma and flavour. Australian Journal of grape and wine research 11: 139-173.

Taylor, M.W., Tsai, P., Anfang, N., Ross, H.A., and Goddard, M.R. (2014) Pyrosequencing reveals regional differences in fruit-associated fungal communities. Environmental microbiology 16: 2848-2858.

Tedersoo, L., Bahram, M., Põlme, S., Kõljalg, U., Yorou, N.S., Wijesundera, R. et al. (2014) Global diversity and geography of soil fungi. science 346: 1256688.

Toju, H., Peay, K.G., Yamamichi, M., Narisawa, K., Hiruma, K., Naito, K. et al. (2018) Core microbiomes for sustainable agroecosystems. Nature Plants 4: 247-257.

Turner, T.R., James, E.K., and Poole, P.S. (2013) The plant microbiome. Genome biology 14: 209.

Van Leeuwen, C., and Seguin, G. (2006) The concept of terroir in viticulture. Journal of Wine Research 17: 110 .

Wagner, M.R., Lundberg, D.S., Coleman-Derr, D., Tringe, S.G., Dangl, J.L., and Mitchell - Olds, T. (2014) Natural soil microbes alter flowering phenology and the intensity of selection on flowering time in a wild Arabidopsis relative. Ecology letters 17: 717-726.

Webb, L., Whetton, P., Bhend, J., Darbyshire, R., Briggs, P., and Barlow, E. (2012) Earlier wine-grape ripening driven by climatic warming and drying and management practices. Nature Climate Change 2: 259. Zarraonaindia, I., Owens, S.M., Weisenhorn, P., West, K., Hampton-Marcell, J., Lax, S. et al. (2015) The soil microbiome influences grapevine-associated microbiota. MBio 6: e02527-02514.

Zhalnina, K., Louie, K.B., Hao, Z., Mansoori, N., da Rocha, U.N., Shi, S. et al. (2018) Dynamic root exudate chemistry and microbial substrate preferences drive patterns in rhizosphere microbial community assembly. Nature microbiology 3: 470-480. 


\section{Table and figure legends}

Table 1 Experimental factors predicting $\alpha$ - and $\beta$-diversity of fungal communities in the vineyard

Fig. 1 Fungal communities demonstrate different structures and compositions depending on habitats in the vineyard. (A) Fungal OTUs shared among soil and grapevine habitats (roots, leaves, flowers, grapes) in the vineyard; (B) Microbial community composition characterised to the genus level (relative abundance $>1.0 \%$ shown); (C) Principal coordinate analysis (PCoA) among plant organs and soil samples at harvest stage based on Bray-Curtis distances; (4) Linear discriminant analysis (LDA) effect size (LEfSe) taxonomic cladogram identifying significantly discriminant taxa associated with habitats at harvest.

Fig. 2 Grape developmental stage influences microbial diversity in the vineyard. (A) $\alpha$-diversity (Shannon index) changes during the growing season (bars indicate SE); (B) Bray-Curtis distance PCoA of grape fungal communities according to the developmental stage. Shown are grapes sampled from fruit set, veraison, and harvest stage. Flowering was excluded as flowers were sampled and counted as another grapevine habitat.

Fig. 3 The core microbiome in grapevine habitats and the soil over the growing season. (A) Percentage (left) and relative abundance (right) of OTUs representing the core versus the remaining microbiome associated with the grapevine (flowers, grapes, leaves, roots); (B) Relative abundance (mean \pm SE) of dominant OTUs across plant habitats; (C - F) Relative abundance changes of dominant fungal genera of grapes (C), leaves (D), roots (E), and soil (F) from the beginning to the end of growing season. 
Fig. 4 The core microbiome (as OTUs) can distinguish developmental stages for plant habitats and soil. Shown are the important features (values > 0.10) based on Mean Decrease Gini (MDG) of random forest models. Core members were coloured in pink (grape), green (leaf), blue (root), and navy (soil). Identified OTU species are given in supplementary table S1.

Fig. 5 Statistically significant and strong co-occurrence relationships among grapevine-associated and soil dominant fungi and weather conditions during annual growth cycle. Network plots for grape (A), leaf (B), and root (C) core microbiota with weather matrices and soil core microbiota. Circle nodes represent core OTUs and environmental variables, with different colours. Direct connections between nodes indicate strong correlations (Spearman correlation coefficient, $\rho \geq$ $0.8 ; \mathrm{p}<0.01$ ). The colour of edges describes the positive correlation (pink) or the negative correlation (grey). The size of nodes is proportioned to the interconnected degree. The width of edges is proportioned to the correlation coefficients. Abbreviations: Env1, solar radiation; Env2, mean high temperature; Env3, mean low temperature; Env4, mean temperature; Env5, precipitation; Env6, evaporation; Env7, transpiration; Env8, relative soil moisture.

\section{Supplementary table and figure legends}

Table S1 OTUs identified as core community associated with the grapevine or within each vineyard habitat

Table S2 Classification of random forest models of the development stage

Table S3 Important features of random forest models outside the core community 
Table S4 ANOVA results of weather conditions between developmental stages

Table S5 Spray programme for the studied vineyards from shoot growth until pre-veraison

Table S6 Soil properties of each sampling site at harvest

Fig. S1 Co-occurrence pattern between the soil core microbiome and weather parameters. Circle nodes represent soil core OTUs and environmental variables, with different colours. Direct connections between nodes indicate strong correlations (Spearman correlation coefficient, $\rho \geq$ $0.8 ; \mathrm{p}<0.01$ ). The colour of edges describes the positive correlation (pink) or the negative correlation (grey). The size of nodes is proportioned to the interconnected degree. The width of edges is proportioned to the correlation coefficients. Abbreviations: Env1, solar radiation; Env2, mean high temperature; Env3, mean low temperature; Env4, mean temperature; Env5, precipitation; Env6, evaporation; Env7, transpiration; Env8, relative soil moisture.

Fig. S2 Experimental design and sampling scheme. (A) Sampling map of two vineyards in Mornington Peninsula wine region, Australia. Five Vitis vinifera Pinot Noir vines were selected in each vineyard. (B) Soils, roots, leaves, and grapes/ flowers were collected in each grapevine throughout the annual growth cycle: flowering, fruit set, veraison (berry colour change), and harvest. Calendar dates of sample collection are indicated for each developmental stage. Grapevines were created with BioRender: https://app.biorender.com/. 
Table 1 Experimental factors predicting $\alpha$ - and $\beta$-diversity of fungal communities in the vineyard

\begin{tabular}{|c|c|c|c|c|c|c|}
\hline \multirow{2}{*}{ Factor } & \multirow{2}{*}{ Sample Group } & \multicolumn{3}{|c|}{$\alpha$-diversity (Shannon) ${ }^{1}$} & \multicolumn{2}{|c|}{$\beta$-diversity (Bray-Curt } \\
\hline & & Df & $\mathrm{F}$ & $p$ & $\mathrm{R}^{2}$ & $p$ \\
\hline Habitat & All stages & 4,155 & 5.206 & 1.05E-02 & 0.271 & $\overline{0.001}$ \\
\hline & Flowering & 3,36 & 18.109 & 4.00E-11 & 0.366 & $\mathbf{0 . 0 0 1}$ \\
\hline & Fruit set & 3,36 & 38.109 & 4.00E-11 & 0.385 & 0.001 \\
\hline & Veraison & 3,36 & 5.668 & 2.93E-03 & 0.466 & $\mathbf{0 . 0 0 1}$ \\
\hline & Harvest & 3,36 & 22.848 & 2.27E-08 & 0.350 & 0.001 \\
\hline Stage & Soil & 3,36 & 3.858 & 0.018 & 0.229 & 0.001 \\
\hline & Root & 3,37 & 1.390 & 0.026 & 0.155 & 0.008 \\
\hline & Leaf & 3,38 & 3.396 & 0.001 & 0.120 & 0.01 \\
\hline & Grape & 2,27 & 6.392 & 2.23E-02 & 0.426 & $\mathbf{0 . 0 0 1}$ \\
\hline Vineyard & Soil & 1,38 & 3.969 & 0.055 & 0.034 & 0.203 \\
\hline & Root & 1,38 & 5.878 & 0.008 & 0.052 & 0.073 \\
\hline & Leaf & 1,38 & 0.018 & 0.894 & 0.017 & $0.80 €$ \\
\hline & Flower & 1,8 & 0.988 & 0.353 & 0.513 & 0.757 \\
\hline & Grape & 1,28 & 0.788 & 0.382 & 0.025 & 0.551 \\
\hline
\end{tabular}

${ }^{1}$ One-way ANOVA

${ }^{2}$ Permutational multivariate analysis of variance (PERMANOVA) using distance matrices Bold values indicate statistically significant results, $p<0.05$ 


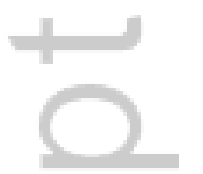

4 
A

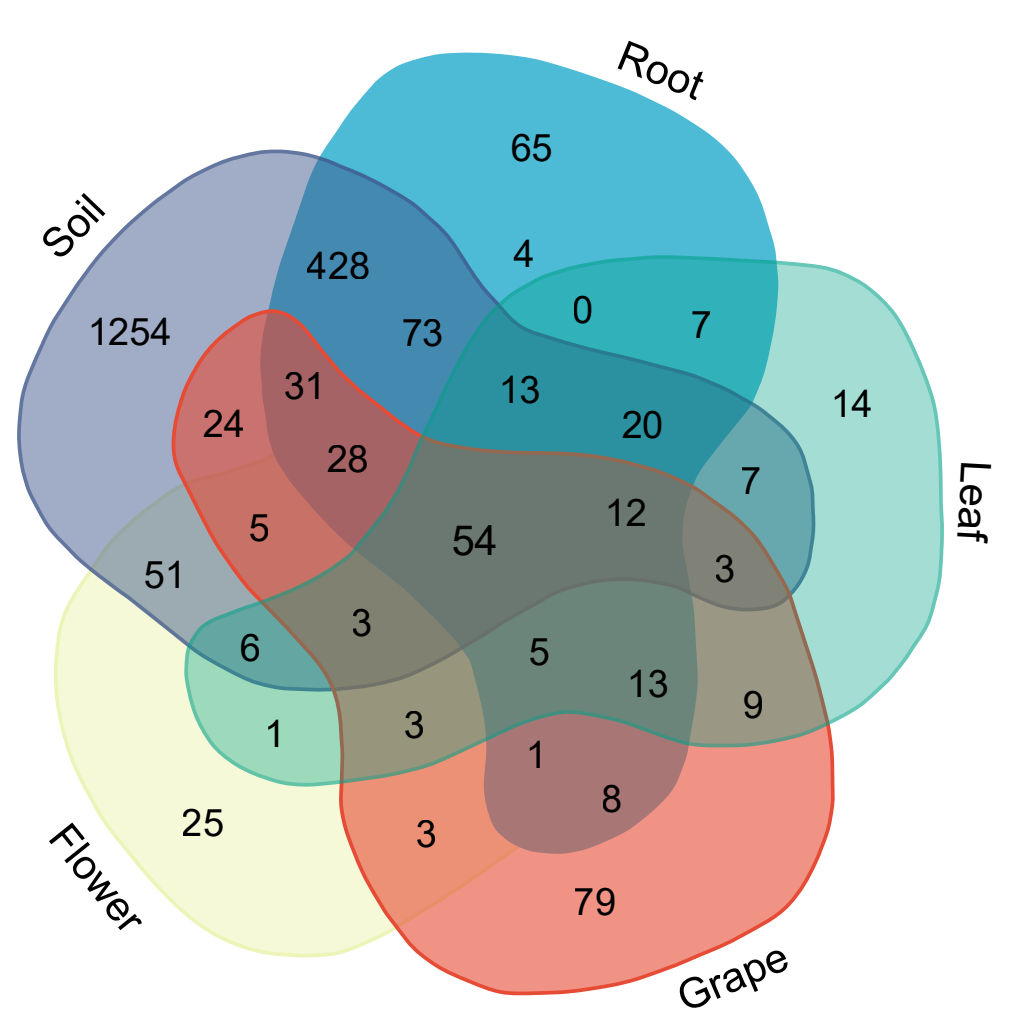

Proportion of OTUs
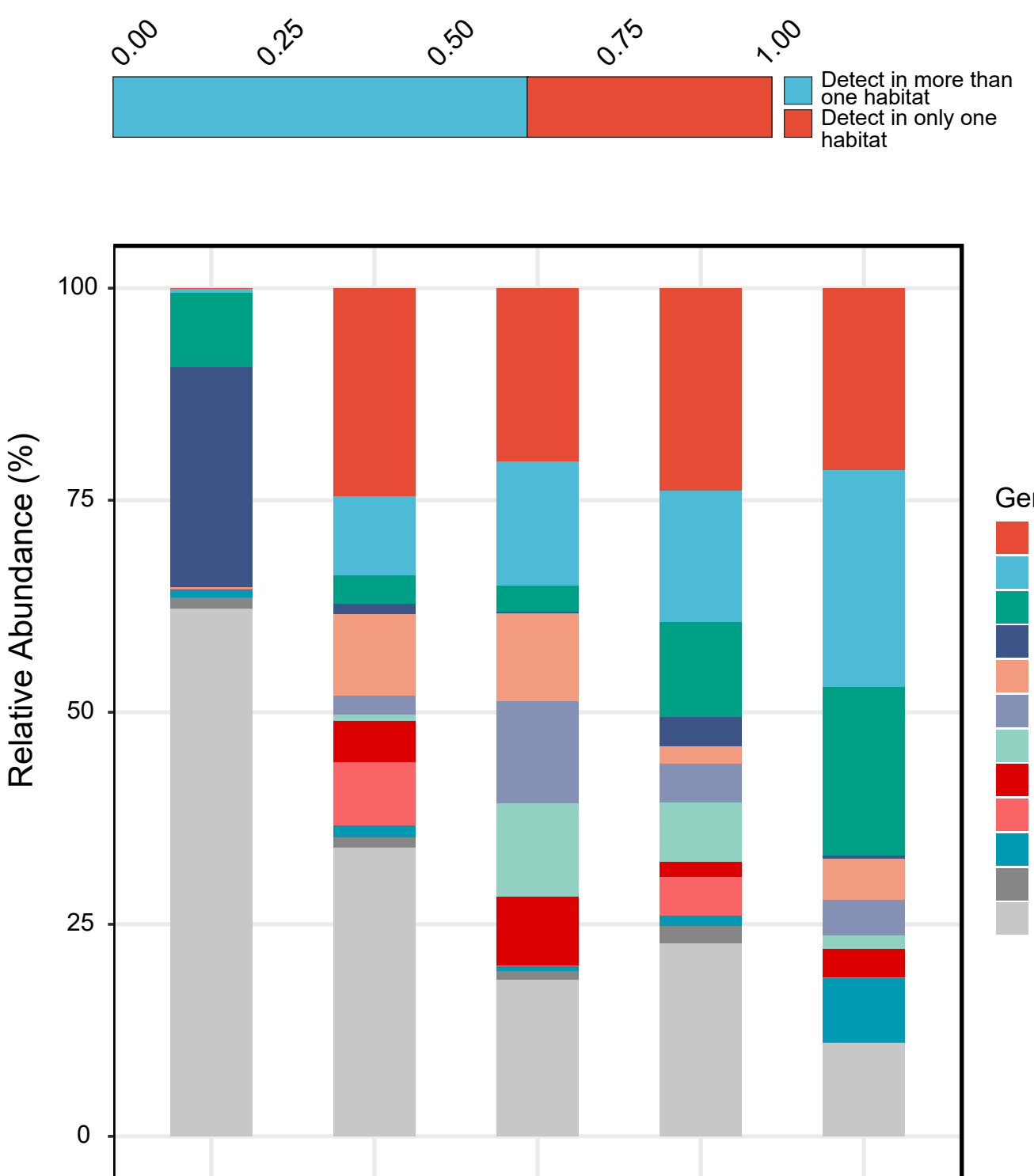

Soil $\begin{gathered}\text { Root Leaf } \\ \text { Flower }\end{gathered}$

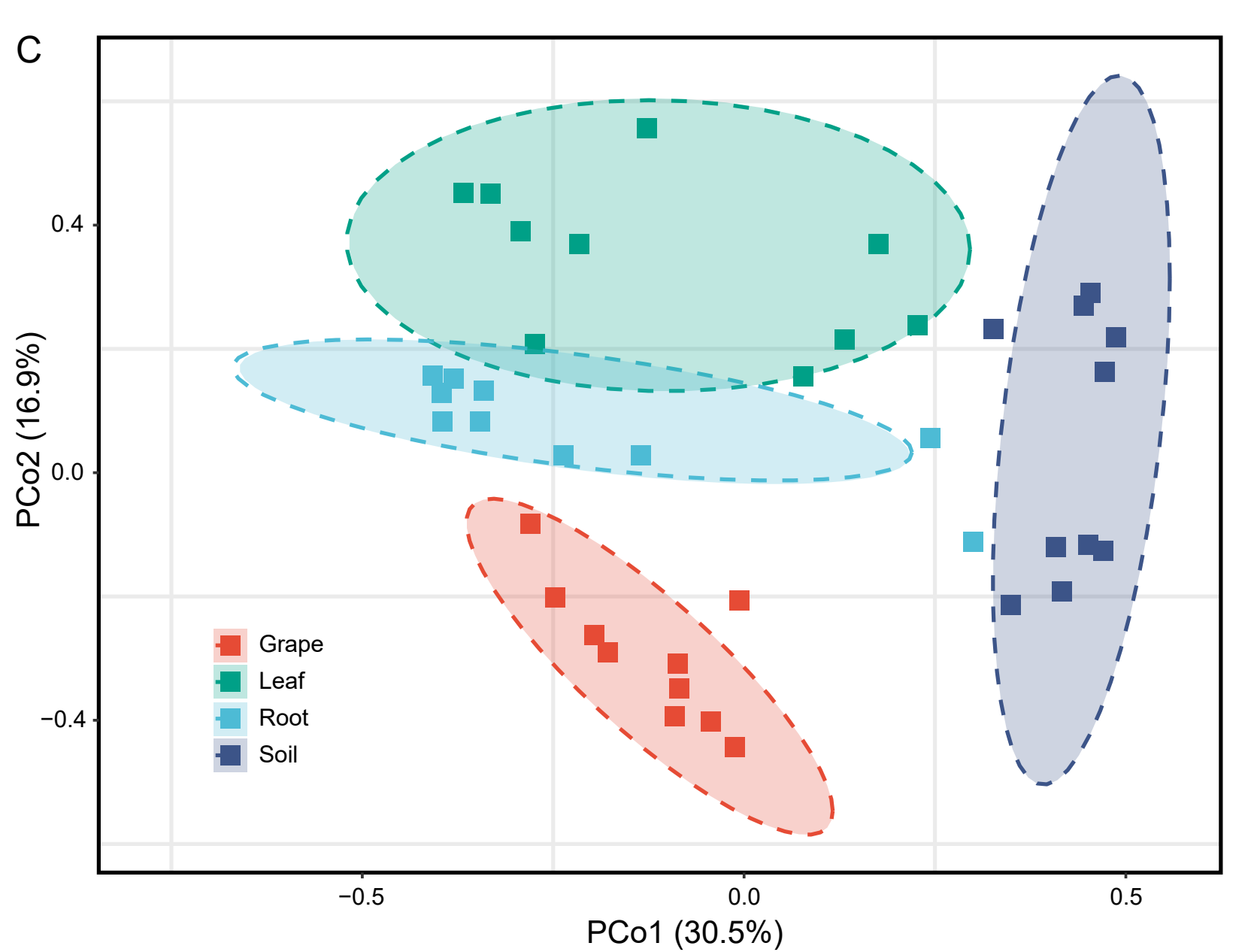

D

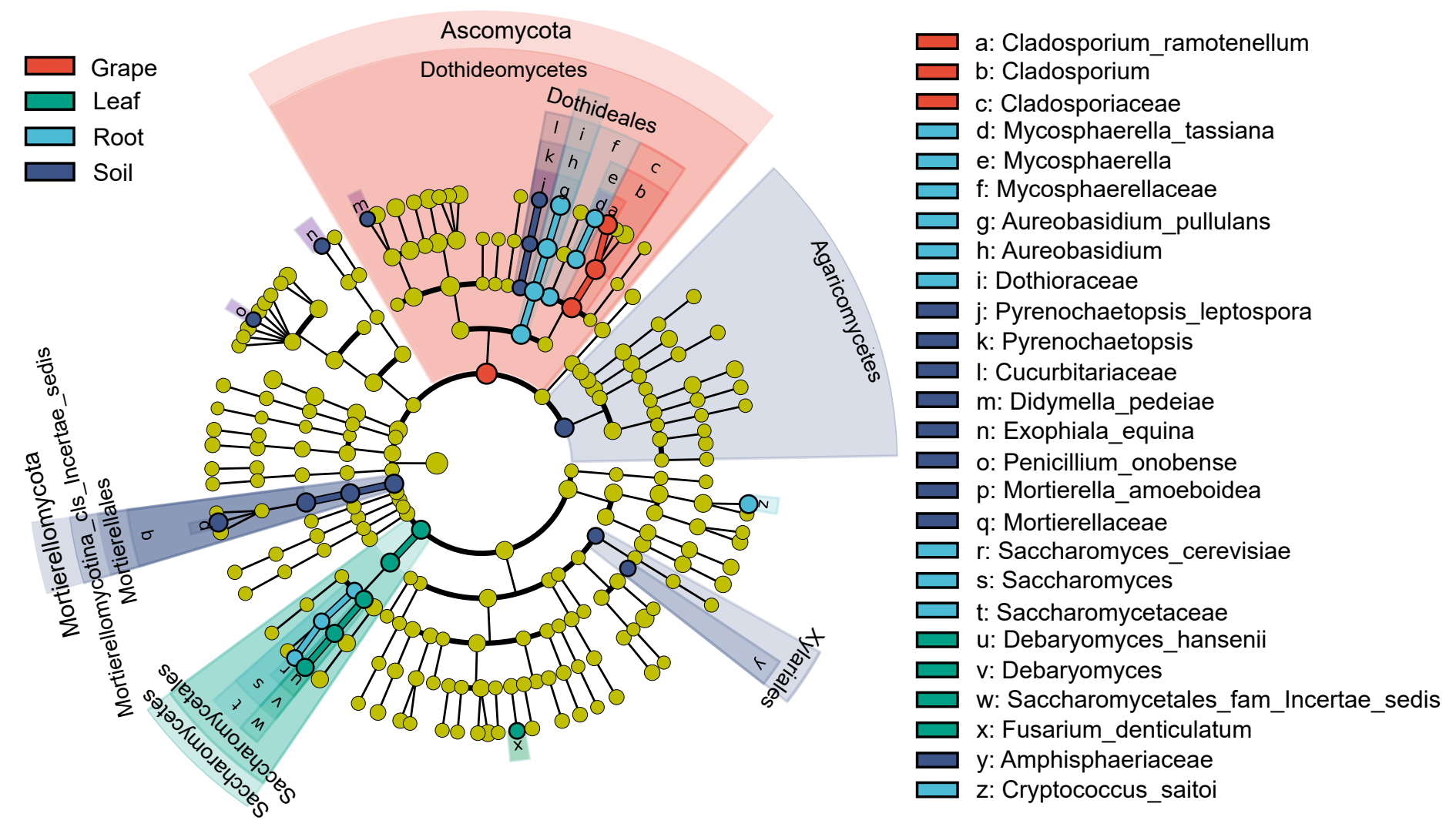

$$
\square \text { Soil }
$$

$\square$ z: Cryptococcus_saito
Genus

Aureobasidium Cladosporium

Epicoccum

Mortierella

Cryptococcus

Debaryomyces

Saccharomyces

Mycosphaerella

Lophiostoma

Alternaria

Penicillium

Others 
A

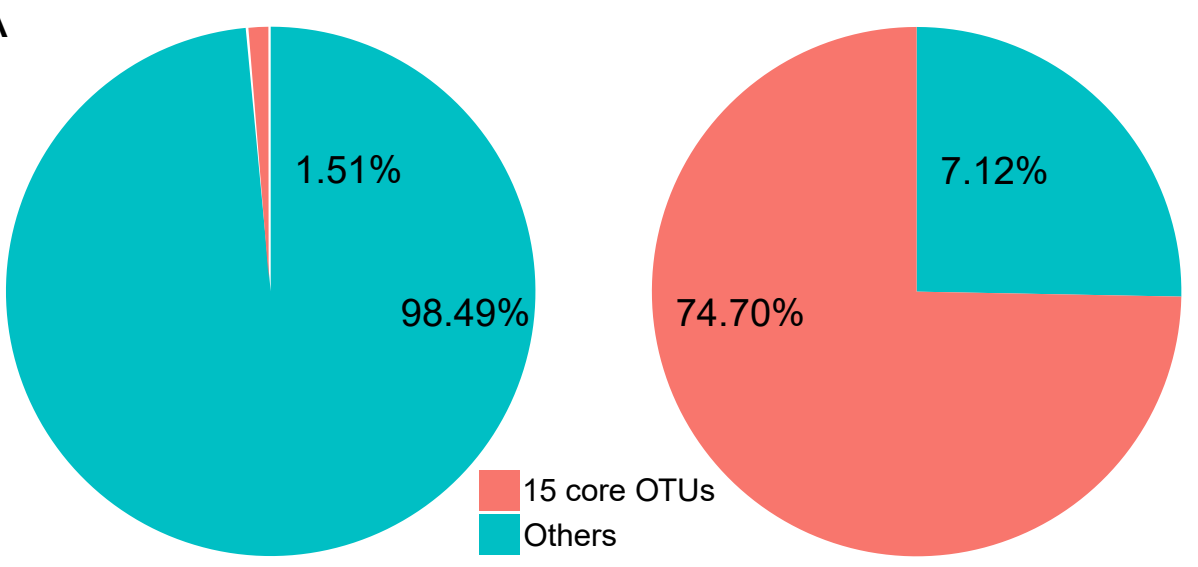

C

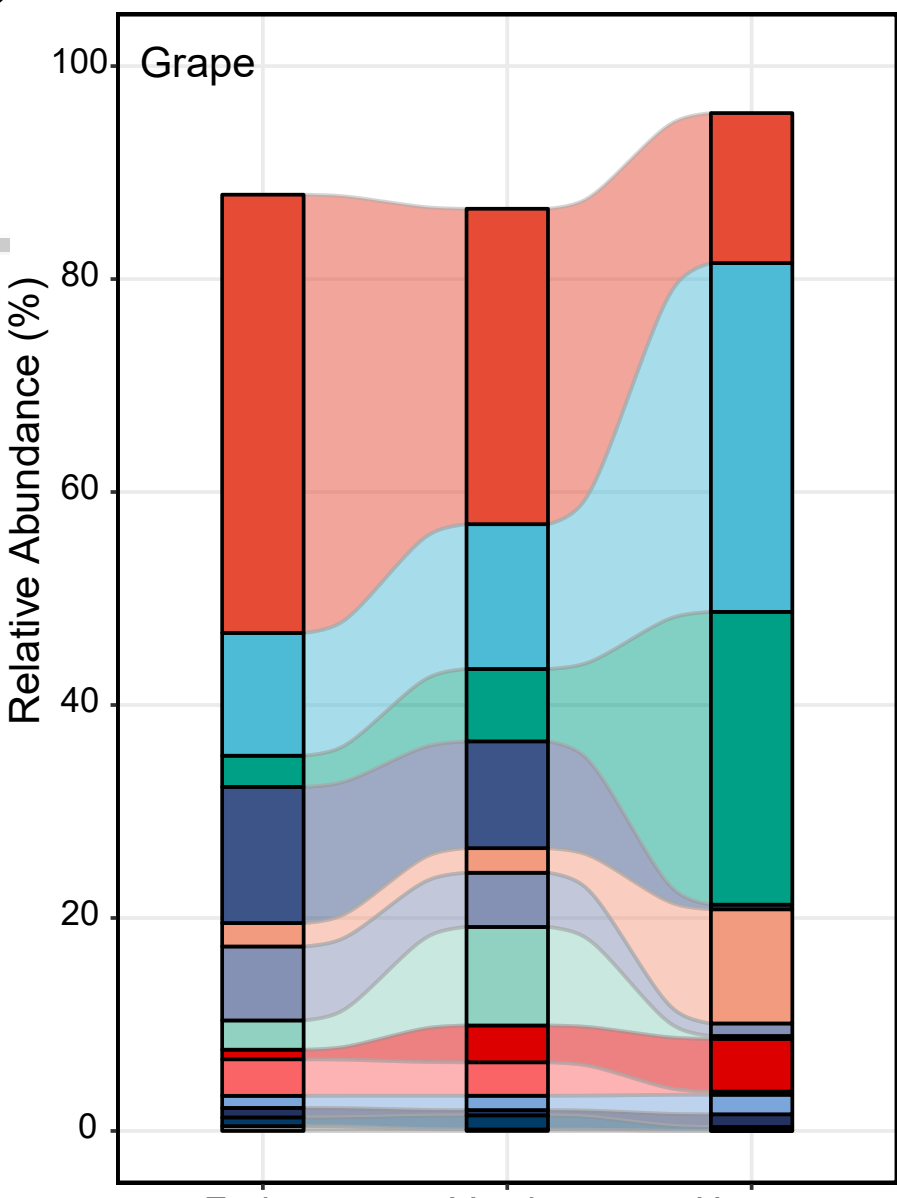

E

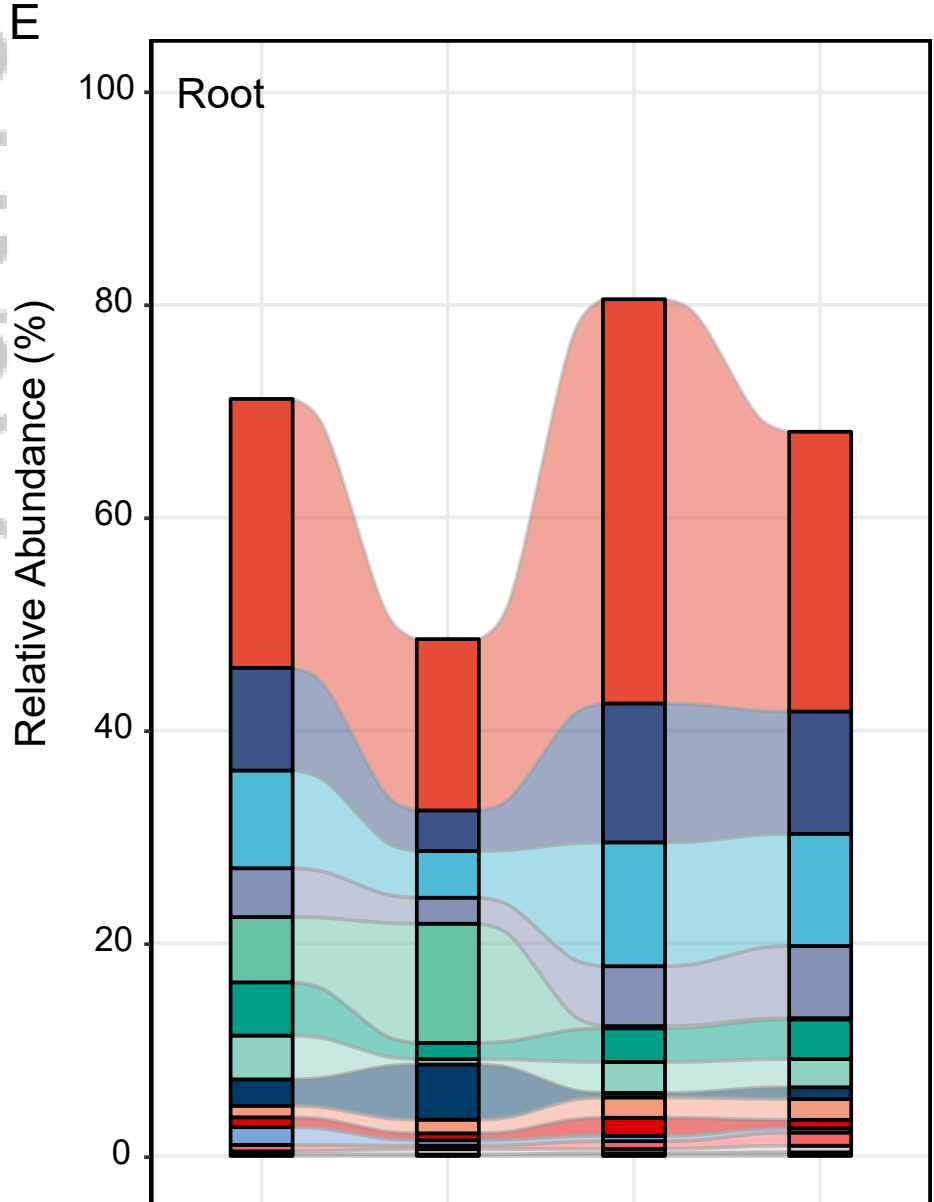

Flowering Fruit set Veraison Harvest
B

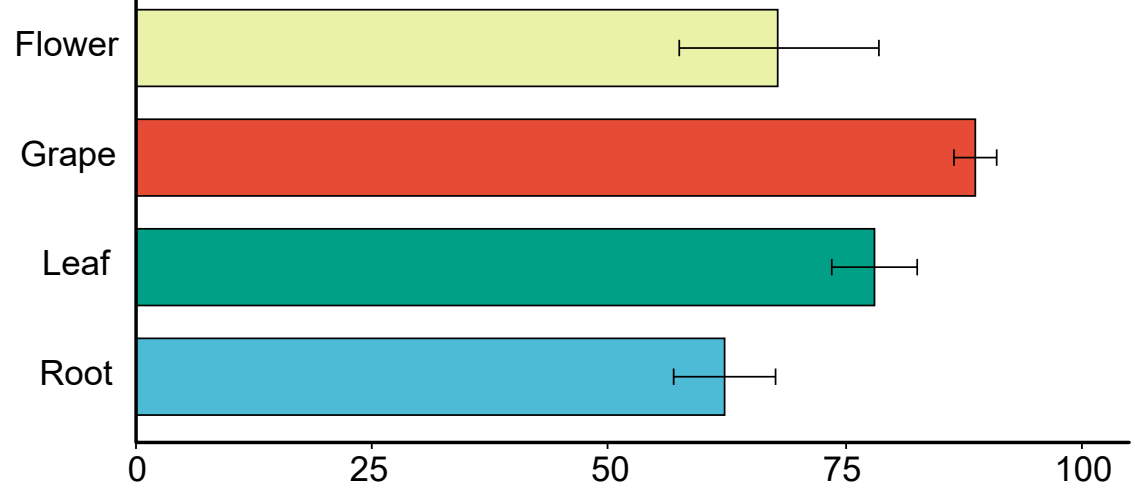

D
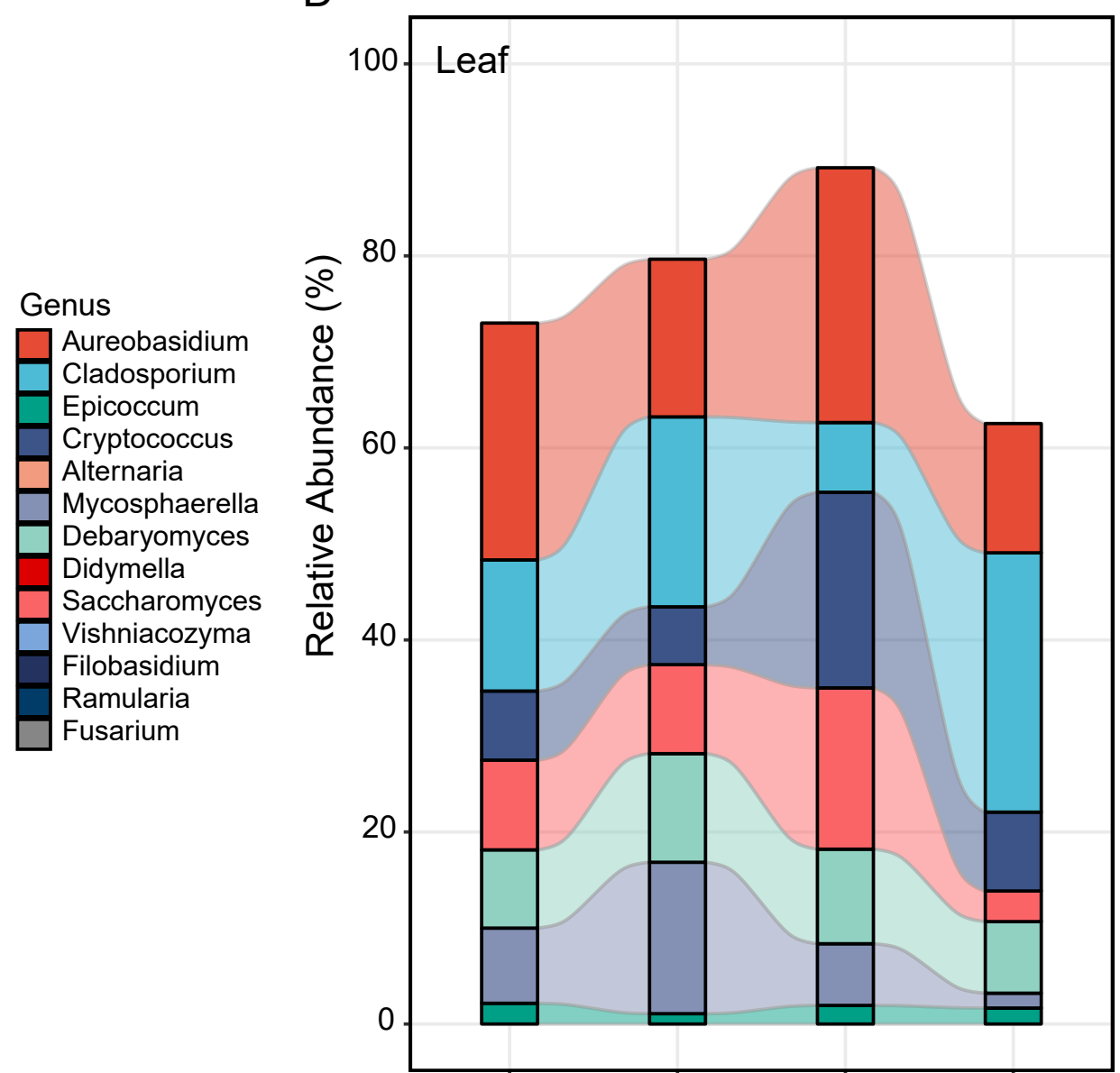

Flowering Fruit set Veraison Harvest

$\mathrm{F}$

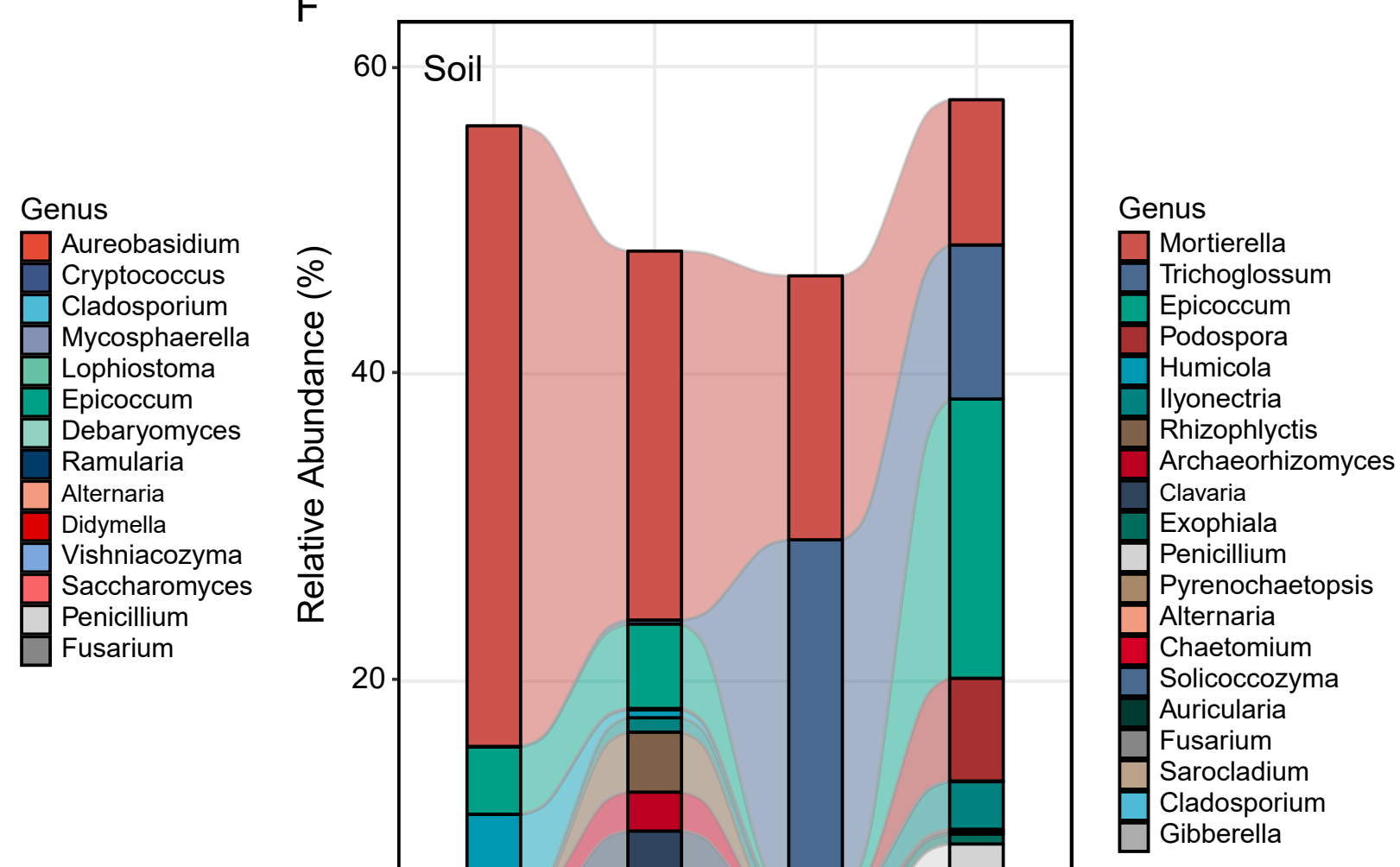

Genus

Aureobasidium

Cladosporium

Cryptococcus

Saccharomyces

Debaryomyces

Mycosphaerella

Epicoccum

This article is protected by copyright. All rights reserved. 
A

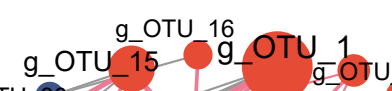
SOTU.8.

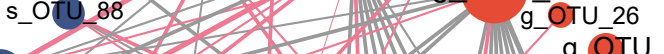

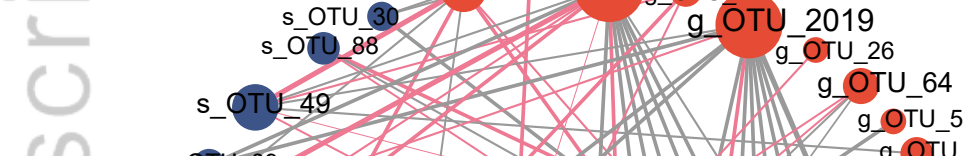

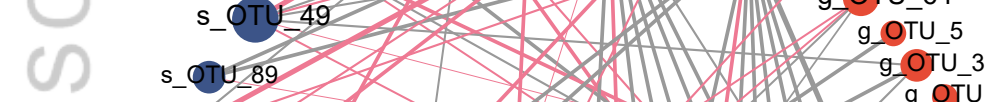
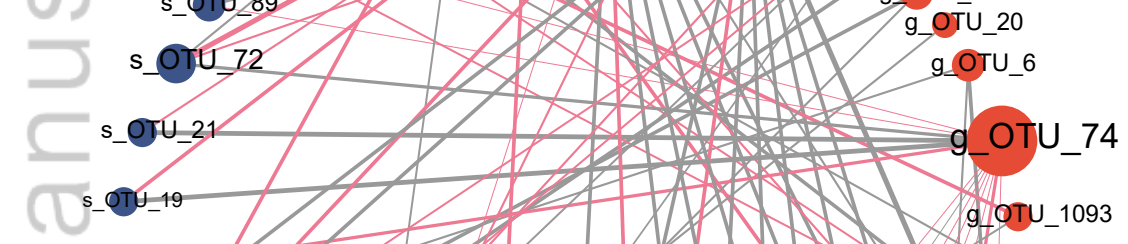

(1) s_ø s_OT-185 s_aTU_150 s_ớu 9

s_QTU_105 185
150
U_91
कTU_105
s_QTU_35
s_aTU_1 1
s_01

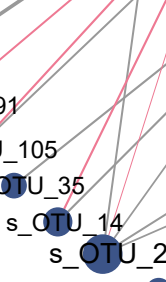
SEDV_23 Env2 g_oTu_9 gLOTU_8

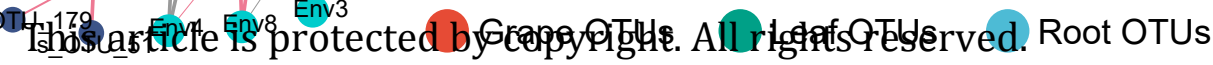

C
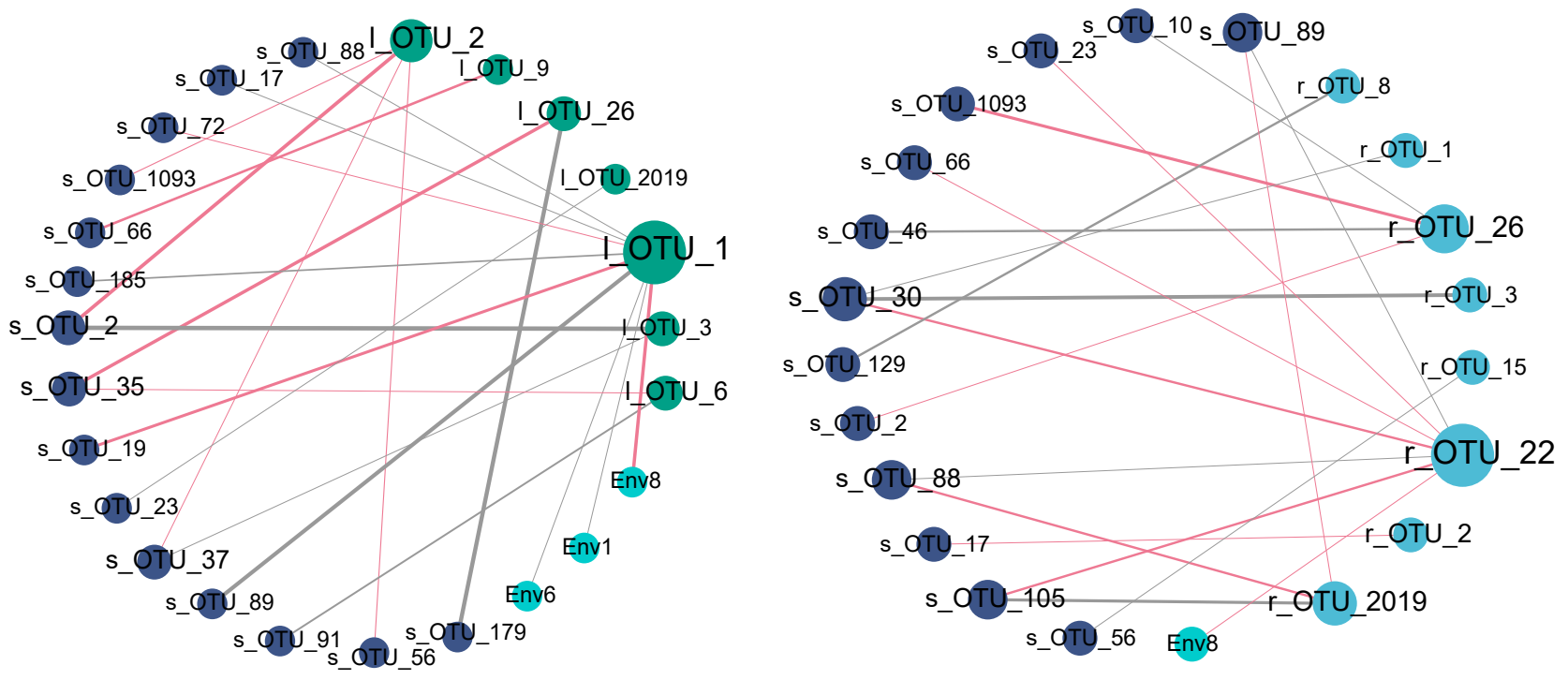

Environmental factors 


\section{University Library}

\section{- M M N E R VA A gateway to Melbourne's research publications}

Minerva Access is the Institutional Repository of The University of Melbourne

Author/s:

Liu, D; Howell, K

Title:

Community succession of the grapevine fungal microbiome in the annual growth cycle

Date:

2020-08-23

Citation:

Liu, D. \& Howell, K. (2020). Community succession of the grapevine fungal microbiome in the annual growth cycle. ENVIRONMENTAL MICROBIOLOGY, 23 (4), pp.1842-1857. https:// doi.org/10.1111/1462-2920.15172.

Persistent Link:

http://hdl.handle.net/11343/276181 